\title{
BEAUVILLE SURFACES, MODULI SPACES AND FINITE GROUPS
}

\author{
SHELLY GARION, MATTEO PENEGINI
}

\begin{abstract}
In this paper we give the asymptotic growth of the number of connected components of the moduli space of surfaces of general type corresponding to certain families of Beauville surfaces with group either $\operatorname{PSL}(2, p)$, or an alternating group, or a symmetric group or an abelian group. We moreover extend these results to regular surfaces isogenous to a higher product of curves.
\end{abstract}

\section{INTRODUCTION}

1.1. Beauville surfaces and surfaces isogenous to a higher product. A surface $S$ is isogenous to a higher product of curves if it is a quotient $\left(C_{1} \times C_{2}\right) / G$, where $C_{1}$ and $C_{2}$ are curves of genus at least two, and $G$ is a finite group acting freely on $C_{1} \times C_{2}$.

In [Cat00] it has been proved that any surface isogenous to a higher product has a unique minimal realization as a quotient $\left(C_{1} \times C_{2}\right) / G$, where $G$ is a finite group acting freely and with the property that no element acts trivially on one of the two factors $C_{i}$. We shall then work only with minimal realizations.

We have two cases: the mixed case where the action of $G$ exchanges the two factors (and then $C_{1}$ and $C_{2}$ are isomorphic), and the unmixed case where $G$ acts diagonally on their product.

We shall use the standard notation in surface theory. We denote by $p_{g}:=h^{0}\left(S, \Omega_{S}^{2}\right)$ the geometric genus of $S, q:=h^{0}\left(S, \Omega_{S}^{1}\right)$ the irregularity of $S, \chi(S)=1+p_{g}-q$ the holomorphic Euler-Poincaré characteristic, $e(S)$ the topological Euler number, and $K_{S}^{2}$ the self-intersection of the canonical divisor (see e.g. [B]).

A surface $S$ isogenous to a higher product is in particular a minimal surface of general type and the numerical invariants of $S$ are related by the following formulae

$$
K_{S}^{2}=8 \chi(S) \text { and } e(S)=4 \chi(S),
$$

by [Cat00, Theorem 3.4]. Moreover, by [Cat00], the irregularity of these surfaces is computed by

$$
q(S)=g\left(C_{1} / G\right)+g\left(C_{2} / G\right) .
$$

By the above formula (2), a surface $S$ isogenous to a higher product of curves has $q(S)=0$ if and only if the two quotients $C_{i} / G$ are isomorphic to $\mathbb{P}^{1}$. Moreover if both coverings $C_{i} \rightarrow C_{i} / G \cong \mathbb{P}^{1}$ are ramified in exactly 
3 points we call $S$ a Beauville surface. This last condition is equivalent to saying that Beauville surfaces are rigid, i.e. have no nontrivial deformations.

Beauville surfaces were introduced by Catanese in Cat00, inspired by a construction of Beauville (see [B]) After this inspiring paper the interest in Beauville surfaces has been enormously increased, see for example BCG05, BCG06, FG, FGJ, FJ, FMP, GLL, GP, GJT, GM].

In this paper we shall deal only with regular (i.e., with $q=0$ ) surfaces isogenous to a product of unmixed type, clearly the Beauville surfaces are among them.

Working out the definition of surfaces isogenous to a product one sees that there is a pure group theoretical condition which characterizes the groups of such surfaces: the existence of a so-called "ramification structure" (see the discussion in Section 2).

Definition 1.1. Let $G$ be a finite group and $r \geq 3$ an integer.

- An $r$-tuple $T=\left(x_{1}, \ldots, x_{r}\right)$ of elements of $G$ is called a spherical $r$-system of generators of $G$ if $\left\langle x_{1}, \ldots, x_{r}\right\rangle=G$ and $x_{1} \cdot \ldots \cdot x_{r}=1$.

- We say that $T$ is of type $\tau=\left(m_{1}, \ldots, m_{r}\right)$ if the orders of $\left(x_{1}, \ldots, x_{r}\right)$ are respectively $\left(m_{1}, \ldots, m_{r}\right)$.

- Moreover, two spherical $r_{i}$-systems $T_{1}=\left(x_{1}, \ldots, x_{r_{1}}\right)$ and $T_{2}=$ $\left(x_{1}, \ldots, x_{r_{2}}\right)$ are said to be disjoint, if:

$$
\Sigma\left(T_{1}\right) \bigcap \Sigma\left(T_{2}\right)=\{1\},
$$

where

$$
\Sigma\left(T_{i}\right):=\bigcup_{g \in G} \bigcup_{j=0}^{\infty} \bigcup_{k=1}^{r_{i}} g \cdot x_{i, k}^{j} \cdot g^{-1} .
$$

Definition 1.2. Let $3 \leq r_{1}, r_{2} \in \mathbb{N}$ and let $\tau_{1}=\left(m_{1,1}, \ldots, m_{1, r_{1}}\right), \tau_{2}=$ $\left(m_{2,1}, \ldots, m_{2, r_{2}}\right)$ be two sequences of natural numbers such that $m_{i, k} \geq 2$.

$A$ (spherical-) unmixed ramification structure of type $\left(\tau_{1}, \tau_{2}\right)$ and size $\left(r_{1}, r_{2}\right)$ for a finite group $G$, is a pair $\left(T_{1}, T_{2}\right)$ of disjoint spherical systems of generators of $G$, whose types are $\left(\tau_{1}, \tau_{2}\right)$.

An unmixed Beauville structure is an unmixed ramification structure with $r_{1}=r_{2}=3$.

Definition 1.3. A triple $(r, s, t) \in \mathbb{N}^{3}$ is said to be hyperbolic if

$$
\frac{1}{r}+\frac{1}{s}+\frac{1}{t}<1
$$

1.2. Moduli spaces of surfaces. By a celebrated Theorem of Gieseker (see [G]), once the two invariants of a minimal surface $S$ of general type, the self-intersection of the canonical divisor $y:=K_{S}^{2}$ and the holomorphic Euler-Poincaré characteristic $x:=\chi(S)$, are fixed, then there exists a coarse quasiprojective moduli space $\mathcal{M}_{y, x}$ of minimal smooth complex surfaces of general type with these invariants. This space consists of a finite number of connected components which can have different dimensions, see Cat84. The union $\mathcal{M}$ over all admissible pairs of invariants $(y, x)$ of these spaces is called the moduli space of surfaces of general type. 
If $S$ is a smooth minimal surface of general type, which is also regular, we denote by $\mathcal{M}(S)$ the subvariety of $\mathcal{M}_{y, x}$, corresponding to surfaces (orientedly) homeomorphic to $S$. Moreover we shall denote by $\mathcal{M}_{y, x}^{0}$ the subspace of the moduli space corresponding to regular surfaces.

It is known that the number of connected components $\delta(y, x)$ of $\mathcal{M}_{y, x}^{0}$ is bounded from above by a function in $y$, more precisely it follows from Cat92 that $\delta(y, x) \leq c y^{77 y^{2}}$, where $c$ is a positive constant. Hence, the number of components has an exponential upper bound in $K^{2}$.

There are also some results regarding the lower bound. In [Man], for example, a sequence $S_{n}$ of simply connected surfaces of general type was constructed, such that the lower bound for the number of the connected components $\delta\left(S_{n}\right)$ of $\mathcal{M}\left(S_{n}\right)$ is given by

$$
\delta\left(S_{n}\right) \geq y_{n}^{\frac{1}{5} \log y_{n}} .
$$

1.3. The results. The motivation of this paper is to show that using pure group theoretical methods we are able to give the asymptotic growth of the number of connected components of the moduli space of surfaces of general type relative to certain sequences of surfaces. More precisely, we exploit the definition and properties of regular surfaces isogenous to a product of curves and in particular, Beauville surfaces, to reduce the geometric problem of computing the number of connected components into the algebraic one of counting orbits of some group actions, which can be effectively computed.

In Cat00], Catanese studied the moduli space of surfaces isogenous to a higher product of curves (see [Cat00, Theorem 4.14]). As a result, he obtains that the moduli space of regular surfaces isogenous to a higher product with fixed invariants: a finite group $G$ and types $\left(\tau_{1}, \tau_{2}\right)$, consists of a finite number of connected components of $\mathcal{M}$. We remark here that since Beauville surfaces are rigid, a Beauville surface yields an isolated point in the moduli space. A group theoretical method to count the number of these components was given in $[\mathrm{BC}]$. Using this method, we deduce the following Theorems, in which we use the following standard notations.

Notation 1.4. Denote:

- $h(n)=O(g(n))$, if $h(n) \leq c g(n)$ for some constant $c>0$, as $n \rightarrow \infty$.

- $h(n)=\Omega(g(n))$, if $h(n) \geq c g(n)$ for some constant $c>0$, as $n \rightarrow \infty$.

- $h(n)=\Theta(g(n))$, if $c_{1} g(n) \leq h(n) \leq c_{2} g(n)$ for some constants $c_{1}, c_{2}>0$, as $n \rightarrow \infty$.

We shall first consider alternating and symmetric groups.

Theorem 1.5. Let $\tau_{1}=\left(m_{1,1}, \ldots, m_{1, r_{1}}\right)$ and $\tau_{2}=\left(m_{2,1}, \ldots, m_{2, r_{2}}\right)$ be two sequences of natural numbers such that $m_{i, k} \geq 2$ and $\sum_{k=1}^{r_{i}}\left(1-1 / m_{i, k}\right)>2$ for $i=1,2$. Let $h\left(\mathfrak{A}_{n} ; \tau_{1}, \tau_{2}\right)$ be the number of connected components of the moduli space of surfaces isogenous to a product with $q=0$, with the alternating group $\mathfrak{A}_{n}$, and with type $\left(\tau_{1}, \tau_{2}\right)$. Then

$$
\text { (a) } h\left(\mathfrak{A}_{n} ; \tau_{1}, \tau_{2}\right)=\Omega\left(n^{r_{1}+r_{2}}\right),
$$

and moreover,

$$
\text { (b) } h\left(\mathfrak{A}_{n} ; \tau_{1}, \tau_{2}\right)=\Omega\left((\log (\chi))^{r_{1}+r_{2}-\epsilon}\right) \text {. }
$$


where $0<\epsilon \in \mathbb{R}$.

Theorem 1.6. Let $\tau_{1}=\left(m_{1,1}, \ldots, m_{1, r_{1}}\right)$ and $\tau_{2}=\left(m_{2,1}, \ldots, m_{2, r_{2}}\right)$ be two sequences of natural numbers such that $m_{i, k} \geq 2$, at least two of $\left(m_{i, 1}, \ldots, m_{i, r_{i}}\right)$ are even and $\sum_{k=1}^{r_{i}}\left(1-1 / m_{i, k}\right)>2$, for $i=1,2$. Let $h\left(\mathfrak{S}_{n} ; \tau_{1}, \tau_{2}\right)$ be the number of connected components of the moduli space of surfaces isogenous to a product with $q=0$, with the symmetric group $\mathfrak{S}_{n}$, and with type $\left(\tau_{1}, \tau_{2}\right)$. Then

and moreover,

$$
\text { (a) } h\left(\mathfrak{S}_{n} ; \tau_{1}, \tau_{2}\right)=\Omega\left(n^{r_{1}+r_{2}}\right)
$$

$$
\text { (b) } h\left(\mathfrak{S}_{n} ; \tau_{1}, \tau_{2}\right)=\Omega\left((\log (\chi))^{r_{1}+r_{2}-\epsilon}\right) .
$$

where $0<\epsilon \in \mathbb{R}$.

The proofs of part (a) of both Theorems are presented in Section 3.2 , and are based on results of Liebeck and Shalev [LS. The proofs of part (b) of both Theorems appear in Section 2.2. In the special case of Beauville surfaces we immediately deduce the following.

Corollary 1.7. Let $\tau_{1}=\left(r_{1}, s_{1}, t_{1}\right)$ and $\tau_{2}=\left(r_{2}, s_{2}, t_{2}\right)$ be two hyperbolic types and let $h\left(\mathfrak{A}_{n} ; \tau_{1}, \tau_{2}\right)$ be the number of Beauville surfaces with the alternating group $\mathfrak{A}_{n}$ and with types $\left(\tau_{1}, \tau_{2}\right)$. Then

$$
\text { (a) } h\left(\mathfrak{A}_{n} ; \tau_{1}, \tau_{2}\right)=\Omega\left(n^{6}\right),
$$

and moreover,

$$
\text { (b) } h\left(\mathfrak{A}_{n} ; \tau_{1}, \tau_{2}\right)=\Omega\left((\log (\chi))^{6-\epsilon}\right) .
$$

where $0<\epsilon \in \mathbb{R}$.

Corollary 1.8. Let $\tau_{1}=\left(r_{1}, s_{1}, t_{1}\right)$ and $\tau_{2}=\left(r_{2}, s_{2}, t_{2}\right)$ be two hyperbolic types, assume that at least two of $\left(r_{1}, s_{1}, t_{1}\right)$ are even and at least two of $\left(r_{2}, s_{2}, t_{2}\right)$ are even, and let $h\left(\mathfrak{S}_{n} ; \tau_{1}, \tau_{2}\right)$ be the number of Beauville surfaces with the symmetric group $\mathfrak{S}_{n}$ and with types $\left(\tau_{1}, \tau_{2}\right)$. Then

$$
\text { (a) } h\left(\mathfrak{S}_{n} ; \tau_{1}, \tau_{2}\right)=\Omega\left(n^{6}\right),
$$

and moreover,

$$
\text { (b) } h\left(\mathfrak{S}_{n}, \tau_{1}, \tau_{2}\right)=\Omega\left((\log (\chi))^{6-\epsilon}\right)
$$

where $0<\epsilon \in \mathbb{R}$.

For the group $\operatorname{PSL}(2, p)$ we obtain the following.

Theorem 1.9. Let $\tau_{1}$ and $\tau_{2}$ be two hyperbolic triples, let $p$ be an odd prime, and consider the group $\operatorname{PSL}(2, p)$. Let $h\left(\operatorname{PSL}(2, p) ; \tau_{1}, \tau_{2}\right)$ be the number of Beauville surfaces with group $\operatorname{PSL}(2, p)$ and with types $\left(\tau_{1}, \tau_{2}\right)$. Then there exists a constant $c=c\left(\tau_{1}, \tau_{2}\right)$, which depends only on the types and not on $p$, such that

$$
h\left(\operatorname{PSL}(2, p) ; \tau_{1}, \tau_{2}\right) \leq c .
$$

The proof of this Theorem is presented in Section 3.3 (see also Remark 2.13).

The following is a natural generalization of the results of [BCG05] regarding abelian groups. 
Theorem 1.10. Let $\left\{S_{p}\right\}$ be the family of surfaces isogenous to a product with $q=0$ and with group $G_{p}:=(\mathbb{Z} / p \mathbb{Z})^{r}$ admitting a ramification structure of type $\tau_{p}=(p, \ldots, p)$ ( $p$ appears $(r+1)$-times) where $p$ is prime. If we denote by $h\left(G_{p} ; \tau_{p}, \tau_{p}\right)$ the number of connected components of the moduli space of isomorphism classes of surfaces isogenous to a product with $q=0$ admitting these data, then

$$
h\left(G_{p} ; \tau_{p}, \tau_{p}\right)=\Theta\left(\chi^{r}\left(S_{p}\right)\right) .
$$

Therefore, there exist families of surfaces such that the degree of the polynomial $h$ in $\chi$ (and so in $K^{2}$ ) can be arbitrarily large. The proof of this Theorem appears in Section 2.2. In the special case of Beauville surfaces we immediately deduce the following.

Corollary 1.11. Let $\left\{S_{p}\right\}$ be the family of Beauville surfaces with $G_{p}:=$ $(\mathbb{Z} / p \mathbb{Z})^{2}$ admitting a ramification structure of type $\tau_{p}=(p, p, p)$ where $p \geq 5$ is prime. If we denote by $h\left(G_{p} ; \tau_{p}, \tau_{p}\right)$ the number of Beauville surfaces admitting these data, then

$$
h\left(G_{p} ; \tau_{p}, \tau_{p}\right)=\Theta\left(\chi^{2}\left(S_{p}\right)\right) .
$$

Acknowledgement. The authors are grateful to Fritz Grunewald for inspiring and motivating us to work on this problem together. He is deeply missed.

The authors would like to thank Ingrid Bauer and Fabrizio Catanese for suggesting the problems, for many useful discussions and for their helpful suggestions. We are grateful to Moshe Jarden and Martin Kassabov for interesting discussions. We also would like to thank G. Jones, G. GonzalesDiez and D. Torres-Teigell for pointing out some subtleties in our first draft.

The authors acknowledge the support of the DFG Forschergruppe 790 "Classification of algebraic surfaces and compact complex manifolds". The first author acknowledges the support of the European Post-Doctoral Institute and the Max-Planck-Institute for Mathematics in Bonn.

\section{From Geometry to Group Theory and Back}

2.1. Ramification structures and Hurwitz components. The study of surfaces isogenous to a higher product is strictly linked to the study of branched coverings of complex curves. We shall recall Riemann's existence theorem which translates the geometric problem of constructing branch coverings into a group theoretical problem.

Definition 2.1. Let $g^{\prime}, m_{1}, \ldots, m_{r}$ be positive integers. An orbifold surface group of type $\left(g^{\prime} \mid m_{1}, \ldots, m_{r}\right)$ is a group presented as follows:

$$
\begin{aligned}
\Gamma\left(g^{\prime} \mid m_{1}, \ldots, m_{r}\right):=\left\langle a_{1}, b_{1}, \ldots, a_{g^{\prime}}, b_{g^{\prime}}, c_{1}, \ldots, c_{r}\right| \\
\left.c_{1}^{m_{1}}=\cdots=c_{r}^{m_{r}}=\prod_{k=1}^{g^{\prime}}\left[a_{k}, b_{k}\right] c_{1} \cdot \ldots \cdot c_{r}=1\right\rangle .
\end{aligned}
$$

If $g^{\prime}=0$ it is called a polygonal group, if $g^{\prime}=0$ and $r=3$ it is called a triangle group. 
We remark that an orbifold surface group is in particular cases a Fuchsian group (see e.g. $[\mathrm{Br}$ and $[\mathrm{LS}]$ ).

The following is a reformulation of Riemann's existence theorem:

Theorem 2.2. A finite group $G$ acts as a group of automorphisms on some compact Riemann surface $C$ of genus $g$ if and only if there are natural numbers $g^{\prime}, m_{1}, \ldots, m_{r}$, and an orbifold homomorphism

$$
\theta: \Gamma\left(g^{\prime} \mid m_{1}, \ldots, m_{r}\right) \rightarrow G
$$

such that $\operatorname{ord}\left(\theta\left(c_{i}\right)\right)=m_{i} \forall i$ and such that the Riemann - Hurwitz relation holds:

$$
2 g-2=|G|\left(2 g^{\prime}-2+\sum_{i=1}^{r}\left(1-\frac{1}{m_{i}}\right)\right) .
$$

If this is the case, then $g^{\prime}$ is the genus of $C^{\prime}:=C / G$. The $G$-cover $C \rightarrow C^{\prime}$ is branched in $r$ points $p_{1}, \ldots, p_{r}$ with branching indices $m_{1}, \ldots, m_{r}$, respectively.

We obtain that the datum of a surface isogenous to a higher product of unmixed type $S=\left(C_{1} \times C_{2}\right) / G$ with $q=0$ is determined, once we look at the monodromy of each covering of $\mathbb{P}^{1}$, by the datum of a finite group $G$ together with two respective disjoint spherical $r_{i}$-systems of generators $T_{1}:=\left(x_{1}, \ldots, x_{r_{1}}\right)$ and $T_{2}:=\left(x_{1}, \ldots, x_{r_{2}}\right)$, such that the types of the systems satisfy (5) with $g^{\prime}=0$ and respectively $g=g\left(C_{i}\right)$. The condition of being disjoint ensures that the action of $G$ on the product of the two curves $C_{1} \times C_{2}$ is free. We remark here that this can be specialized to $r_{i}=3$, and therefore can be used to construct Beauville surfaces. This description gives at once the Definition 1.2.

Remark 2.3. Note that a group $G$ and an unmixed ramification structure (or equivalently a Beauville structure) for it determine the main invariants of the surface $S$. Indeed, as a consequence of the Zeuthen-Segre formula one has:

$$
e(S)=4 \frac{\left(g\left(C_{1}\right)-1\right)\left(g\left(C_{2}\right)-1\right)}{|G|} .
$$

Hence, by (11) and (5) we obtain:

$$
\left.\left.4 \chi(S)=4\left(1+p_{g}\right)=|G| \cdot\left(-2+\sum_{k=1}^{r_{1}}\left(1-\frac{1}{m_{1, k}}\right)\right)\right) \cdot\left(-2+\sum_{k=1}^{r_{2}}\left(1-\frac{1}{m_{2, k}}\right)\right)\right),
$$

and so, in the Beauville case,

$$
4 \chi(S)=4\left(1+p_{g}\right)=|G|\left(1-\mu_{1}\right)\left(1-\mu_{2}\right),
$$

where

$$
\mu_{i}:=\frac{1}{m_{i, 1}}+\frac{1}{m_{i, 2}}+\frac{1}{m_{i, 3}}, \quad(i=1,2) .
$$

Now, it is left to verify that indeed $g\left(C_{1}\right) \geq 2$ and $g\left(C_{2}\right) \geq 2$. This follows from Equation (5) and from the following Lemma. 
Lemma 2.4. Let $G$ be a finite non-trivial group and $\left(T_{1}, T_{2}\right)$ a spherical unmixed ramification structure of $G$ of size $\left(r_{1}, r_{2}\right)$, then

$$
\mathbb{Z} \ni \frac{|G|\left(-2+\sum_{l=1}^{r_{i}}\left(1-\frac{1}{m_{i, l}}\right)\right)}{2}+1 \geq 2, \text { for } i=1,2 .
$$

The fact that the number in (9) is an integer follows from Riemann's existence theorem. We need to prove that this integer is at least 2 , namely that $\sum_{l=1}^{r_{i}}\left(1-\frac{1}{m_{i, l}}\right)>2$ for $i=1,2$. We shall give two proofs for this fact, a geometric one and a group theoretic one (in Section 3.4.4), both are based on results of Bauer, Catanese and Grunewald.

Geometrical proof. Let $S=\left(C_{1} \times C_{2}\right) / G$ be a surface isogenous to a product with $q(S)=0$, notice first that $g\left(C_{1}\right) \neq 1$.

Indeed, suppose that $g\left(C_{1}\right)=1$, then $S \rightarrow C_{2} / G \cong \mathbb{P}^{1}$ is an elliptic fibration with fibre isomorphic to $C_{1}$ or to a multiple of $C_{1}$. Since $C_{1}$ is an elliptic curve, the Zeuthen-Segre Theorem holds in the following form:

$$
e(S)=4\left(g\left(C_{1}\right)-1\right)\left(g\left(C_{2} / G\right)-1\right)=0 .
$$

Since $S$ is isogenous to a product $4 \chi(S)=e(S)=0$, but we have $\chi(S)=$ $1+p_{g}-q=1+p_{g}>0$. Hence $g\left(C_{1}\right) \neq 1$.

Second, suppose that $S$ is a $\mathbb{P}^{1}$-bundle. Then $S$ cannot be non-rational, because non-rational ruled surfaces have $q>0$. Hence $S$ must be rational. If $S$ is rational then $p_{g}=0$, and surfaces with $p_{g}=q=0$ isogenous to a product were classified by Bauer-Catanese-Grunewald in [BCG08], and the only rational one is $S=\mathbb{P}^{1} \times \mathbb{P}^{1}$, therefore $G$ is trivial and this case is also excluded.

Let $S$ be a surface isogenous to a higher product of unmixed type with $q=0$, and with group $G$ and a pair of two disjoint spherical $r_{i}$-systems of generators of types $\left(\tau_{1}, \tau_{2}\right)$. By (77) we have $\chi(S)=\chi\left(G,\left(\tau_{1}, \tau_{2}\right)\right)$, and consequentially, by (11), $K_{S}^{2}=K^{2}\left(G,\left(\tau_{1}, \tau_{2}\right)\right)=8 \chi(S)$.

Let us fix a group $G$ and a pair of unmixed ramification types $\left(\tau_{1}, \tau_{2}\right)$, and denote by $\mathcal{M}_{\left(G,\left(\tau_{1}, \tau_{2}\right)\right)}$ the moduli space of isomorphism classes of surfaces isogenous to a product admitting these data, by Cat00 the space $\mathcal{M}_{\left(G,\left(\tau_{1}, \tau_{2}\right)\right)}$ consists of a finite number of connected components. Indeed, there is a group theoretical procedure to count these components, which is described in $[\mathrm{BC}]$.

Definition 2.5. The braid group of the sphere $\mathbf{B}_{r}:=\pi_{0}\left(\operatorname{Diff}\left(\mathbb{P}^{1}-\left\{p_{1}, \ldots, p_{r}\right\}\right)\right)$ operates on the epimorphism $\theta$ defined in (4):

$$
\pi_{1}\left(\mathbb{P}^{1}-\left\{p_{1}, \ldots, p_{r}\right\}\right) /\left\langle\gamma^{m_{1}}, \ldots, \gamma^{m_{r}}\right\rangle \cong \Gamma:=\Gamma\left(0 \mid m_{1}, \ldots, m_{r}\right) \stackrel{\theta}{\longrightarrow} G .
$$

Indeed, if $\sigma \in \mathbf{B}_{r}$ then the operation is given by $\theta \circ \sigma$. The orbits of this action are called Hurwitz equivalence classes of the spherical systems of generators.

Definition 2.6. Let $G$ be a finite group, let $r \geq 3$ and $2 \leq m_{1} \leq m_{2} \leq$ $\cdots \leq m_{r}$ be integers. Assume that $T=\left(x_{1}, \ldots, x_{r}\right)$ is a spherical $r$-system of generators of $G$. 
- We say that $T$ has an unordered type $\tau$ if the orders of $\left(x_{1}, \ldots, x_{r}\right)$ are $\left(m_{1}, \ldots, m_{r}\right)$ up to a permutation, namely, if there is a permutation $\pi \in \mathfrak{S}_{r}$ such that

$$
\operatorname{ord}\left(x_{1}\right)=m_{\pi(1)}, \ldots, \operatorname{ord}\left(x_{r}\right)=m_{\pi(r)} .
$$

- We shall denote:

$$
\mathcal{S}(G, \tau):=\{\text { spherical } r \text {-systems for } G \text { of type } \tau\} .
$$

Notation 2.7. Let $\left(T_{1}, T_{2}\right)$ be a pair of disjoint spherical $r_{i}$-systems of generators of type $\left(\tau_{1}, \tau_{2}\right)$, we call the pair $\left(T_{1}, T_{2}\right)$ unordered if $T_{1}$ and $T_{2}$ have unordered types $\tau_{1}$ and $\tau_{2}$ respectively.

We shall denote by $\mathcal{U}\left(G ; \tau_{1}, \tau_{2}\right)$ the set of all unordered pairs $\left(T_{1}, T_{2}\right)$ of disjoint spherical $r_{i}$-systems of generators of type $\left(\tau_{1}, \tau_{2}\right)$.

Theorem 2.8. [BC, Theorem 1.3]. Let $S$ be a surface isogenous to a higher product of unmixed type and with $q=0$. Then to $S$ we attach its finite group $G$ (up to isomorphism) and the equivalence classes of an unordered pair of disjoint spherical systems of generators $\left(T_{1}, T_{2}\right)$ of $G$, under the equivalence relation generated by:

(i) Hurwitz equivalence for $T_{1}$;

(ii) Hurwitz equivalence for $T_{2}$;

(iii) Simultaneous conjugation for $T_{1}$ and $T_{2}$, i.e., for $\phi \in \operatorname{Aut}(G)$ we let $\left(T_{1}:=\left(x_{1,1}, \ldots, x_{r_{1}, 1}\right), \quad T_{2}:=\left(x_{1,2}, \ldots, x_{r_{2}, 2}\right)\right)$ be equivalent to

$$
\left(\phi\left(T_{1}\right):=\left(\phi\left(x_{1,1}\right), \ldots, \phi\left(x_{r_{1}, 1}\right)\right), \quad \phi\left(T_{2}\right):=\left(\phi\left(x_{1,2}\right), \ldots, \phi\left(x_{r_{2}, 2}\right)\right)\right) .
$$

Then two surfaces $S, S^{\prime}$ are deformation equivalent if and only if the corresponding equivalence classes of pairs of spherical generating systems of $G$ are the same.

Recall that

Lemma 2.9. [V], Lemma 9.4]. The inner automorphism group, $\operatorname{Inn}(G)$, leaves each braid orbit invariant.

This Lemma allows us to use the above Theorem of Bauer and Catanese also for non-abelian groups, although the original statement was given only for abelian groups.

Once we fix a finite group $G$ and a pair of types $\left(\tau_{1}, \tau_{2}\right)$ (of size $\left(r_{1}, r_{2}\right)$ ) of an unmixed ramification structure for $G$, counting the number of connected components of $\mathcal{M}_{\left(G,\left(\tau_{1}, \tau_{2}\right)\right)}$ is then equivalent to the group theoretical problem of counting the number of classes of pairs of spherical systems of generators of $G$ of type $\left(\tau_{1}, \tau_{2}\right)$ under the equivalence relation given in the following definition (see e.g. [BCG08, §1.1]).

Definition 2.10. Denote by $h\left(G ; \tau_{1}, \tau_{2}\right)$ the number of Hurwitz components, namely the number of orbits of $\mathcal{U}\left(G ; \tau_{1}, \tau_{2}\right)$ under the following actions:

$$
\begin{aligned}
& \text { if } \tau_{1} \neq \tau_{2} \text { : the action of }\left(\mathbf{B}_{r_{1}} \times \mathbf{B}_{r_{2}}\right) \times \operatorname{Aut}(G) \text {, given by: } \\
& \qquad\left(\left(\gamma_{1}, \gamma_{2}\right), \phi\right) \cdot\left(T_{1}, T_{2}\right):=\left(\phi\left(\gamma_{1}\left(T_{1}\right)\right), \phi\left(\gamma_{2}\left(T_{2}\right)\right)\right), \\
& \text { where } \gamma_{1} \in \mathbf{B}_{r_{1}}, \gamma_{2} \in \mathbf{B}_{r_{2}}, \phi \in \operatorname{Aut}(G) \text { and }\left(T_{1}, T_{2}\right) \in \mathcal{U}\left(G ; \tau_{1}, \tau_{2}\right) .
\end{aligned}
$$


if $\tau_{1}=\tau_{2}$ : the action of $\left(\mathbf{B}_{r} \prec \mathbb{Z} / 2 \mathbb{Z}\right) \times \operatorname{Aut}(G)$, where $\mathbb{Z} / 2 \mathbb{Z}$ acts on $\left(T_{1}, T_{2}\right)$ by exchanging the two factors.

In case of Beauville surfaces we define $h$ as above substituting $r_{1}$ and $r_{2}$ with 3.

2.2. Counting connected components in the moduli space. In this Section we prove Theorems 1.5(b), 1.6(b) and 1.10. We start by proving a more general statement regarding families of finite groups.

Proposition 2.11. Fix $r_{1}$ and $r_{2}$ in $\mathbb{N}$. Let $\left\{G_{n}\right\}_{n=1}^{\infty}$ be a family of finite groups, which admit an unmixed ramification structure of size $\left(r_{1}, r_{2}\right)$. Let $\tau_{n, 1}=\left(m_{n, 1,1}, \ldots, m_{n, 1, r_{1}}\right)$ and $\tau_{n, 2}=\left(m_{n, 2,1}, \ldots, m_{n, 2, r_{2}}\right)$ be sequences of types $\left(\tau_{n, 1}, \tau_{n, 2}\right)$ of unmixed ramification structures for $G_{n}$, and $\left\{S_{n}\right\}_{n=1}^{\infty}$ be the family of surfaces isogenous to higher product with $q=0$ admitting the given data, then as $\left|G_{n}\right| \stackrel{n \rightarrow \infty}{\longrightarrow} \infty$ :

(i) $\chi\left(S_{n}\right)=\Theta\left(\left|G_{n}\right|\right)$.

(ii) $h\left(G_{n} ; \tau_{n, 1}, \tau_{n, 2}\right)=O\left(\chi\left(S_{n}\right)^{r_{1}+r_{2}-2}\right)$.

Proof. $\quad$ (i) Note that, for $i=1,2$,

$$
\frac{1}{42} \leq-2+\sum_{j=1}^{r_{i}}\left(1-\frac{1}{m_{n, i, j}}\right) \leq r_{i}-2
$$

Indeed, for $r_{i}=3$, the minimal value for $\left(1-\mu_{i}\right)$ is $1 / 42$. For $r_{i}=4$, the minimal value for $\left(-2+\sum_{j=1}^{r_{i}}\left(1-\frac{1}{m_{n, i, j}}\right)\right)$ is $1 / 6$, and when $r_{i} \geq 5$, this value is at least $1 / 2$.

Now, by Equation (77),

$$
4 \chi\left(S_{n}\right)=\left|G_{n}\right| \cdot\left(-2+\sum_{j=1}^{r_{1}}\left(1-\frac{1}{m_{n, 1, j}}\right)\right) \cdot\left(-2+\sum_{j=1}^{r_{2}}\left(1-\frac{1}{m_{n, 2, j}}\right)\right),
$$

hence

$$
\frac{\left|G_{n}\right|}{4 \cdot 42^{2}} \leq \chi\left(S_{n}\right) \leq \frac{\left(r_{1}-2\right)\left(r_{2}-2\right)\left|G_{n}\right|}{4} .
$$

(ii) For $i=1,2$, any spherical $r_{i}$-system of generators $T_{n, i}$ contains at most $r_{i}-1$ independent elements of $G_{n}$. Thus, the size of the set of all unordered pairs of type $\left(\tau_{n, 1}, \tau_{n, 2}\right)$ is bounded from above, by

$$
\left|\mathcal{U}\left(G_{n} ; \tau_{n, 1}, \tau_{n, 2}\right)\right| \leq\left|G_{n}\right|^{r_{1}+r_{2}-2},
$$

and so, the number of connected components is bounded from above by

$$
h\left(G_{n} ; \tau_{n, 1}, \tau_{n, 2}\right) \leq\left|G_{n}\right|^{r_{1}+r_{2}-2} .
$$

Now, the result follows from $(i)$.

By taking $r_{1}=r_{3}=3$ we get the following Corollary.

Corollary 2.12. Let $\left\{G_{n}\right\}_{n=1}^{\infty}$ be a family of finite groups, which admit an unmixed Beauville structure. Let $\tau_{n, 1}=\left(m_{n, 1,1}, m_{n, 1,2}, m_{n, 1,3}\right)$ and $\tau_{n, 2}=$ $\left(m_{n, 2,1}, m_{n, 2,2}, m_{n, 2,3}\right)$ be sequences of types $\left(\tau_{n, 1}, \tau_{n, 2}\right)$ of unmixed Beauville structures for $G_{n}$, and let $\left\{S_{n}\right\}_{n=1}^{\infty}$ be the family of Beauville surfaces admitting the given data, then as $\left|G_{n}\right| \stackrel{n \rightarrow \infty}{\longrightarrow} \infty$ : 
(i) $\chi\left(S_{n}\right)=\Theta\left(\left|G_{n}\right|\right)$.

(ii) $h\left(G_{n} ; \tau_{n, 1}, \tau_{n, 2}\right)=O\left(\chi\left(S_{n}\right)^{4}\right)$.

With the calculation done in this paper we can give a more accurate description of the asymptotic growth of $h$ in case of Beauville surfaces and surfaces isogenous to a higher product with $q=0$, for certain families of finite groups.

Remark 2.13. Let $\tau_{1}=(r, r, r)$ and $\tau_{2}=(s, s, s)$ be two hyperbolic types, where $r$ and $s$ are two distinct primes which are strictly larger than 5 . By [GP], there exist infinitely many primes $p$ for which the group $\operatorname{PSL}(2, p)$ admits an unmixed Beauville structure of type $\left(\tau_{1}, \tau_{2}\right)$.

Let us consider the corresponding Beauville surfaces $S_{p}$, then by Proposition 2.11, as $p \rightarrow \infty$ :

$$
\chi\left(S_{p}\right)=\Theta\left(p^{3}\right),
$$

while, by Theorem 1.9 we have

$$
h\left(\operatorname{PSL}(2, p), \tau_{1}, \tau_{2}\right)<c,
$$

where $c=c\left(\tau_{1}, \tau_{2}\right)$ is a constant which depends only on the types and not on $p$. Namely, there exists an infinite family of surfaces for which $h$ remains bounded while $\chi$ grows to infinity.

On the other hand, when considering the groups $\mathfrak{A}_{n}$ and $\mathfrak{S}_{n}$ one obtains the following lower bound.

Proof of Theorem 1.5(b) and 1.6(b). Let $\left\{S_{n}\right\}$ be a family of surfaces isogenous to a higher product with $q=0$, with group either $\mathfrak{A}_{n}$ or $\mathfrak{S}_{n}$, and $\tau_{1}$ and $\tau_{2}$ are two types which satisfy the assumptions of the Theorems. Then by Proposition 2.11, as $n \rightarrow \infty$ :

$$
\chi\left(S_{n}\right)=\Theta(n !),
$$

while, by Theorems 1.5(a) and 1.6(a),

$$
\begin{aligned}
h\left(\mathfrak{A}_{n}, \tau_{1}, \tau_{2}\right) & =\Omega\left(n^{r_{1}+r_{2}}\right), \text { and } \\
h\left(\mathfrak{S}_{n}, \tau_{1}, \tau_{2}\right) & =\Omega\left(n^{r_{1}+r_{2}}\right) .
\end{aligned}
$$

Therefore,

$$
\begin{aligned}
& h\left(\mathfrak{A}_{n}, \tau_{1}, \tau_{2}\right)=\Omega\left(\left(\log \left(\chi\left(S_{n}\right)\right)\right)^{r_{1}+r_{2}-\epsilon}\right) \text { and } \\
& h\left(\mathfrak{S}_{n}, \tau_{1}, \tau_{2}\right)=\Omega\left(\left(\log \left(\chi\left(S_{n}\right)\right)\right)^{r_{1}+r_{2}-\epsilon}\right) .
\end{aligned}
$$

where $0<\epsilon \in \mathbb{R}$.

For abelian groups one can moreover obtain both a lower and an upper bound.

Proof of Theorem 1.10. Consider the family $\left\{S_{p}\right\}$ of surfaces isogenous to a higher product with $q=0$, where $p$ is prime, admitting type $\tau_{p}=(p, \ldots, p)$ $(p$ appears $(r+1)$-times $)$ and group $G_{p}:=(\mathbb{Z} / p \mathbb{Z})^{r}$, then by Proposition 2.11, we have as $p \rightarrow \infty$ :

$$
\chi\left(S_{p}\right)=\Theta\left(p^{r}\right),
$$

while by Proposition 3.26 ,

$$
h\left(G_{p} ; \tau_{p}, \tau_{p}\right)=\Theta\left(p^{r^{2}}\right) .
$$


Therefore,

$$
h\left(G_{p} ; \tau_{p}, \tau_{p}\right)=\Theta\left(\chi^{r}\left(S_{p}\right)\right)
$$

\section{Finite Groups, Ramification Structures and Hurwitz COMPONENTS}

3.1. Braid group actions. Recall that the braid group $\mathbf{B}_{r}$ on $r$ strands can be presented as

$$
\left.\mathbf{B}_{r}=\left\langle\sigma_{1}, \ldots, \sigma_{r-1}\right| \sigma_{i} \sigma_{i+1} \sigma_{i}=\sigma_{i+1} \sigma_{i} \sigma_{i+1}, \sigma_{i} \sigma_{j}=\sigma_{j} \sigma_{i} \text { if }|i-j| \geq 2\right\rangle .
$$

The action of $\mathbf{B}_{r}$ on the set of spherical $r$-systems of generators for $G$ of unordered type $\tau=\left(m_{1}, \ldots, m_{r}\right)$, which was given in Definition 2.5, is given by

$$
\sigma_{i}:\left(x_{1}, \ldots, x_{i}, \ldots, x_{r}\right) \rightarrow\left(x_{1}, \ldots, x_{i-1}, x_{i} x_{i+1} x_{i}^{-1}, x_{i}, x_{i+2} \ldots, x_{r}\right),
$$

for $i=1, \ldots, r-1$.

There is also a natural action of $\operatorname{Aut}(G)$ given by

$$
\phi\left(x_{1}, \ldots, x_{r}\right)=\left(\phi\left(x_{1}\right), \ldots, \phi\left(x_{r}\right)\right), \quad \phi \in \operatorname{Aut}(G) .
$$

Since the two actions of $\mathbf{B}_{r}$ and $\operatorname{Aut}(G)$ commute, one gets a double action of $\mathbf{B}_{r} \times \operatorname{Aut}(G)$ on the set of spherical $r$-systems of generators for $G$ of an unordered type $\tau=\left(m_{1}, \ldots, m_{r}\right)$.

Let $x \in G$ and denote by $C=x^{\operatorname{Aut}(G)}$ the $\operatorname{Aut}(G)$-equivalence class of $x$. Since all the elements in $C$ have the same order, we may define $\operatorname{ord}(C):=$ $\operatorname{ord}(x)$.

Let $\mathbf{C}=\left(C_{1}, \ldots, C_{r}\right)$ be a set of $\operatorname{Aut}(G)$-equivalence classes. Denote

$$
N(\mathbf{C}):=\left\{\left(x_{1}, \ldots, x_{r}\right) \in G^{r}: x_{1} \cdot \ldots \cdot x_{r}=1,\left\langle x_{1}, \ldots, x_{r}\right\rangle=G\right. \text { and }
$$
there is a permutation $\pi \in \mathfrak{S}_{r}$ with $x_{\pi(i)} \in C_{i}$ for all $\left.i\right\}$.

We say that $\mathbf{C}$ has type $\tau=\left(m_{1}, \ldots, m_{r}\right)$ if $N(\mathbf{C}) \neq \emptyset$ and $\operatorname{ord}\left(C_{i}\right)=m_{i}$ (for $i=1, \ldots, r)$. $\mathbf{C}$ has an unordered type $\tau$ if $N(\mathbf{C}) \neq \emptyset$ and the orders of $C_{1}, \ldots, C_{r}$ are $m_{1}, \ldots, m_{r}$ up to a permutation. We denote

$$
N(\tau)=\left\{\mathbf{C}=\left(C_{1}, \ldots, C_{r}\right): \mathbf{C} \text { has an unordered type } \tau\right\} .
$$

Observe that the action of $\mathbf{B}_{r}$ preserves the set $N(\mathbf{C})$, since it preserves the conjugacy classes, and hence the $\operatorname{Aut}(G)$-equivalence classes, of the elements in a spherical $r$-system of generators of $G$. It is clear that the action of $\operatorname{Aut}(G)$ also preserves the set $N(\mathbf{C})$. The following Lemma immediately follows.

Lemma 3.1. Let $\tau_{1}$ and $\tau_{2}$ be two types, then

$$
\begin{gathered}
h\left(G ; \tau_{1}, \tau_{2}\right) \geq \#\left\{\mathbf{C}_{i}, \mathbf{D}_{j}: \mathbf{C}_{i}=\left(C_{i, 1}, \ldots, C_{i, r_{1}}\right) \text { and } \mathbf{D}_{j}=\left(D_{j, 1}, \ldots, D_{j, r_{2}}\right),\right. \\
\text { where } \mathbf{C}_{i} \in N\left(\tau_{1}\right) \text { for all } i, \mathbf{D}_{j} \in N\left(\tau_{2}\right) \text { for all } j \text {, and } \\
\text { the } \left.\operatorname{Aut}(G)-\text { classes }\left\{C_{i, k}\right\}_{i, k} \text { and }\left\{D_{j, l}\right\}_{j, l} \text { are all distinct }\right\} .
\end{gathered}
$$


One can moreover restrict the action of $\operatorname{Aut}(G)$ to the action of $\operatorname{Inn}(G) \cong$ $G$, which is given by

$$
g:\left(x_{1}, \ldots, x_{r}\right) \mapsto\left(g x_{1} g^{-1}, \ldots, g x_{r} g^{-1}\right), \quad g \in G .
$$

Denote by $O_{T}^{G}$ the orbit of $T=\left(x_{1}, \ldots, x_{r}\right)$ under the action of $\operatorname{Inn}(G) \cong$ $G$, and by $O_{T}^{\mathbf{B}_{r}}$ the orbit of $T$ under the action of $\mathbf{B}_{r}$. By Lemma 2.9, the action of $\operatorname{Inn}(G)$ leaves the orbit $O_{T}^{\mathrm{B}}$ invariant, namely,

Lemma 3.2. $O_{T}^{G} \subseteq O_{T}^{\mathbf{B}_{r}}$.

Hence, we get an induced action of $\operatorname{Out}(G)=\operatorname{Aut}(G) / \operatorname{Inn}(G)$ on the set of spherical $r$-systems of generators for $G$ of unordered type $\tau$.

In the special case of $\mathbf{B}_{3}$, the braid group on 3 strands, we show that the inverse inclusion also holds, and one can therefore deduce a more accurate bound on the number of orbits.

Let $T=\left(x, y,(x y)^{-1}\right)$ be a spherical 3 -system of generators for $G$, and let

$$
\begin{aligned}
T^{u n}:=\left(x, y,(x y)^{-1}\right) \cup & \left(y, x,(y x)^{-1}\right) \cup\left(x,(y x)^{-1}, y\right) \\
& \cup\left(y,(x y)^{-1}, x\right) \cup\left((x y)^{-1}, x, y\right) \cup\left((y x)^{-1}, y, x\right),
\end{aligned}
$$

be an unordered spherical 3-system of generators.

Observe that $O_{T^{u n}}^{G}:=\left\{O_{T}^{G}: T \in T^{u n}\right\}$, where

$$
O_{T}^{G}=\left\{\left(g x g^{-1}, g y g^{-1}, g(x y)^{-1} g^{-1}\right): g \in G\right\} .
$$

Lemma 3.3. The action of $\mathbf{B}:=\mathbf{B}_{3}$ preserves $O_{T^{u n}}^{G}$.

Proof. Let $\left(x, y,(x y)^{-1}\right)$ be a spherical 3 -system for $G$, then the action of $\mathbf{B}:=\mathbf{B}_{3}=\left\langle\sigma_{1}, \sigma_{2}\right\rangle$ is given by:

$\sigma_{1}:\left(x, y, y^{-1} x^{-1}\right) \rightarrow\left(x y x^{-1}, x, y^{-1} x^{-1}\right)=x\left(y, x, x^{-1} y^{-1}\right) x^{-1} \in O_{\left(y, x,(y x)^{-1}\right)}^{G}$, and

$$
\sigma_{2}:\left(x, y, y^{-1} x^{-1}\right) \rightarrow\left(x, y y^{-1} x^{-1} y^{-1}, y\right)=\left(x, x^{-1} y^{-1}, y\right) \in O_{\left(x,(y x)^{-1}, y\right)}^{G} .
$$

From Lemma 3.2 and Lemma 3.3 we deduce the following orbit equality.

Corollary 3.4. $O_{T^{u n}}^{G}=O_{T^{u n}}^{\mathbf{B}_{3}}$.

Denote by $d=d(G ; \tau)$ the number of orbits in the set of spherical 3 -systems of generators for $G$ of unordered type $\tau$, under the action of $\mathbf{B}_{3}$. Then by Corollary 3.4 .

Corollary 3.5. $d(G ; \tau)=\#\left\{O_{T^{u n}}^{G}: T \in \mathcal{S}(G, \tau)\right\}$.

Now, one can bound the number of Hurwitz components using $d(G ; \tau)$.

Corollary 3.6. Let $\tau_{1}$ and $\tau_{2}$ be two types, then

$$
\frac{d\left(G ; \tau_{1}\right) \cdot d\left(G ; \tau_{2}\right)}{2|\operatorname{Out}(G)|} \leq h\left(G ; \tau_{1}, \tau_{2}\right) \leq d\left(G ; \tau_{1}\right) \cdot d\left(G ; \tau_{2}\right) .
$$

The proof of the Corollary follows from the following Lemma, which summarizes some well-known facts regarding group actions on finite sets. 
Lemma 3.7. Let $H$ and $K$ be groups and let $X$ and $Y$ be finite sets.

If $H$ acts on $X$ and $x \in X$, we denote $O_{x}^{H}:=\{h \cdot x: h \in H\}$ the orbit of $x$ under the action of $H$ and $\Omega_{H}=\Omega_{H}^{X}$ the set of orbits in $X$.

(i) If the groups $H$ and $K$ act on a set $X$, then for every $x \in X$,

$$
\left|O_{x}^{H}\right| \leq\left|O_{x}^{H \times K}\right| \leq\left|O_{x}^{H}\right| \cdot|K| .
$$

(ii) If the groups $H$ and $K$ act on a set $X$, and there is some positive constant $c$ such that $\left|O_{x}^{H}\right| \leq c\left|O_{x}^{K}\right|$ for any $x \in X$, then

$$
\left|\Omega_{H}\right| \geq \frac{1}{c}\left|\Omega_{K}\right| \text {. }
$$

(iii) If the groups $H$ and $K$ act on the sets $X$ and $Y$ respectively, then

$$
\left|\Omega_{H \times K}^{X \times Y}\right|=\left|\Omega_{H}^{X}\right| \cdot\left|\Omega_{K}^{Y}\right| \cdot
$$

Proof of Corollary [3.6. By Lemma 3.7.

$$
\Omega_{\left(B_{3} \times B_{3}\right) \times \operatorname{Aut}(G)}^{\mathcal{U}\left(G ; \tau_{1}, \tau_{2}\right)} \leq \Omega_{B_{3} \times B_{3}}^{\mathcal{U}\left(G ; \tau_{1}, \tau_{2}\right)}=d\left(G ; \tau_{1}\right) \cdot d\left(G ; \tau_{2}\right) .
$$

On the other direction, by Lemma 3.2, Lemma 3.7 and Definition 2.10, we have

In case $\tau_{1} \neq \tau_{2}$ :

$$
\Omega_{\left(B_{3} \times B_{3}\right) \times \operatorname{Aut}(G)}^{\mathcal{U}\left(G ; \tau_{1}, \tau_{2}\right)}=\Omega_{\left(B_{3} \times B_{3}\right) \times \operatorname{Out}(G)}^{\mathcal{U}\left(G ; \tau_{1}, \tau_{2}\right)} \quad \geq \frac{\Omega_{B_{3} \times B_{3}}^{\mathcal{U}\left(G ; \tau_{1}, \tau_{2}\right)}}{|\operatorname{Out}(G)|}=\frac{d\left(G ; \tau_{1}\right) \cdot d\left(G ; \tau_{2}\right)}{|\operatorname{Out}(G)|} .
$$

In case $\tau_{1}=\tau_{2}=\tau$ :

$$
\Omega_{\left(B_{3} ; \mathbb{Z} / 2 \mathbb{Z}\right) \times \operatorname{Aut}(G)}^{\mathcal{U}(G ; \tau)}=\Omega_{\left(B_{3} \mathbb{Z} / 2 \mathbb{Z}\right) \times \operatorname{Out}(G)}^{\mathcal{U}(G ; \tau, \tau)} \quad \geq \frac{\Omega_{B_{3} \mathbb{Z} / 2 \mathbb{Z}}^{\mathcal{U}(G ; \tau, \tau)}}{|\operatorname{Out}(G)|}=\frac{d(G ; \tau)^{2}}{2|\operatorname{Out}(G)|} .
$$

3.2. Hurwitz components for $\mathfrak{A}_{n}$ and $\mathfrak{S}_{n}$. In this Section we prove Theorems 1.5(a) and 1.6(a) regarding alternating and symmetric groups.

The results of Liebeck and Shalev, which are stated below, are applicable to any Fuchsian group $\Gamma$, however, we shall use them only for the case of orbifold surface groups (see Definition 2.1)

$$
\Gamma=\Gamma\left(g^{\prime} \mid m_{1}, \ldots, m_{r}\right)
$$

that satisfy the inequality

$$
2 g^{\prime}-2+\sum_{i=1}^{r}\left(1-\frac{1}{m_{i}}\right)>0 .
$$

Definition 3.8. Let $C_{i}=g_{i}^{\mathfrak{S}_{n}}(1 \leq i \leq r)$ be conjugacy classes in $\mathfrak{S}_{n}$, and let $m_{i}$ be the order of $g_{i}$. Define $\operatorname{sgn}\left(C_{i}\right)=\operatorname{sgn}\left(g_{i}\right)$, and write $\mathbf{C}=$ $\left(C_{1}, \ldots, C_{r}\right)$. Define

$$
\operatorname{Hom}_{\mathbf{C}}\left(\Gamma, \mathfrak{S}_{n}\right)=\left\{\phi \in \operatorname{Hom}\left(\Gamma, \mathfrak{S}_{n}\right): \phi\left(x_{i}\right) \in C_{i} \text { for } 1 \leq i \leq r\right\} .
$$

Definition 3.9. Conjugacy classes in $\mathfrak{S}_{n}$ of cycle-shape $\left(\mathrm{m}^{k}\right)$, where $n=$ $m k$, namely, containing $k$ cycles of length $m$ each, are called homogeneous. A conjugacy class having cycle-shape $\left(m^{k}, 1^{f}\right)$, namely, containing $k$ cycles of length $m$ each and $f$ fixed points, with $f$ bounded, is called almost homogeneous. 
Theorem 3.10. [LS, Theorem 1.9]. Let $\Gamma$ be a Fuchsian group, and let $C_{i}$ $(1 \leq i \leq r)$ be conjugacy classes in $\mathfrak{S}_{n}$ with cycle-shapes $\left(m_{i}^{k_{i}}, 1^{f_{i}}\right)$, where $f_{i}<f$ for some constant $f$ and $\prod_{i=1}^{r} \operatorname{sgn}\left(C_{i}\right)=1$. Set $\mathbf{C}=\left(C_{1}, \ldots, C_{r}\right)$. Then the probability that a random homomorphism in $\operatorname{Hom}_{\mathbf{C}}\left(\Gamma, \mathfrak{S}_{n}\right)$ has image containing $\mathfrak{A}_{n}$ tends to 1 as $n \rightarrow \infty$.

Using Theorem 3.10, Liebeck and Shalev deduced the following Corollary regarding $\mathfrak{S}_{n}$.

Corollary 3.11. [LS, Theorem 1.10]. Let $\Gamma=\Gamma\left(-\mid m_{1}, \ldots, m_{r}\right)$ be a polygonal group which satisfies the above inequality (10), and assume that at least two of $m_{1}, \ldots, m_{r}$ are even. Then $\Gamma$ surjects to all but finitely many symmetric groups $\mathfrak{S}_{n}$.

As consequences of these results we recall the following theorems of [GP].

Theorem 3.12. Let $\tau_{1}=\left(m_{1,1}, \ldots, m_{1, r_{1}}\right)$ and $\tau_{2}=\left(m_{2,1}, \ldots, m_{2, r_{2}}\right)$ be two sequences of natural numbers such that $m_{i, k} \geq 2$ and $\sum_{k=1}^{r_{i}}\left(1-1 / m_{i, k}\right)>$ 2 for $i=1,2$. Then almost all alternating groups $\mathfrak{A}_{n}$ admit an unmixed ramification structure of type $\left(\tau_{1}, \tau_{2}\right)$.

Theorem 3.13. Let $\tau_{1}=\left(m_{1,1}, \ldots, m_{1, r_{1}}\right)$ and $\tau_{2}=\left(m_{2,1}, \ldots, m_{2, r_{2}}\right)$ be two sequences of natural numbers such that $m_{i, k} \geq 2$, at least two of $\left(m_{i, 1}, \ldots, m_{i, r_{i}}\right)$ are even and $\sum_{k=1}^{r_{i}}\left(1-1 / m_{i, k}\right)>2$, for $i=1,2$. Then almost all symmetric groups $\mathfrak{S}_{n}$ admit an unmixed ramification structure of type $\left(\tau_{1}, \tau_{2}\right)$.

The proofs of the two theorems are based on the following generalization of the algorithm appearing in GP.

Algorithm 3.14. Given two sequences of natural numbers $\tau_{1}=\left(m_{1,1}, \ldots, m_{1, r_{1}}\right)$ and $\tau_{2}=\left(m_{2,1}, \ldots, m_{2, r_{2}}\right)$, such that $m_{i, k} \geq 2$, then one can choose $r_{1}+r_{2}$ almost homogeneous conjugacy classes $C_{m_{1,1}}, \ldots, C_{m_{1, r_{1}}}, C_{m_{2,1}}, \ldots, C_{m_{2, r_{2}}}$ in $\mathfrak{S}_{n}$, of orders $m_{1,1}, \ldots, m_{1, r_{1}}, m_{2,1}, \ldots, m_{2, r_{2}}$ respectively, such that they contain only even permutations, and they all have different numbers of fixed points.

We can now prove Theorems 1.5(a) and 1.6(a).

Proof of Theorem 1.5( $(a)$. Let $\tau_{1}=\left(m_{1,1}, \ldots, m_{1, r_{1}}\right)$ and $\tau_{2}=\left(m_{2,1}, \ldots, m_{2, r_{2}}\right)$ be two sequences of natural numbers such that $m_{j, l} \geq 2$ and $\sum_{l=1}^{r_{j}}(1-$ $\left.1 / m_{j, l}\right)>2$ for $j=1,2$. Let $k \in \mathbb{N}$ be an arbitrary integer, and assume that $n$ is large enough. By slightly modifying Algorithm 3.14, we may actually choose $\left(r_{1}+r_{2}\right) k$ almost homogeneous conjugacy classes in $\mathfrak{S}_{n}$,

$$
\left\{C_{m_{1,1}, i}, \ldots, C_{m_{1, r_{1}}, i}, C_{m_{2,1}, i}, \ldots, C_{m_{2, r_{2}}, i}\right\}_{i=1}^{k},
$$

which contain even permutations, such that every $r_{1}+r_{2}$ classes have orders $m_{1,1}, \ldots, m_{1, r_{1}}, m_{2,1}, \ldots, m_{2, r_{2}}$ respectively, and all the $\left(r_{1}+r_{2}\right) k$ conjugacy classes have different numbers of fixed points.

Hence, if $n$ is large enough, there are $\left(r_{1}+r_{2}\right) k$ different $\mathfrak{S}_{n}$-conjugacy classes in $\mathfrak{A}_{n}$, and moreover, for each $1 \leq i_{1}, \ldots, i_{r_{1}}, j_{1}, \ldots, j_{r_{2}} \leq k$, the conjugacy classes $\left(C_{m_{1,1}, i_{1}}, \ldots, C_{m_{1, r_{1}, i_{r_{1}}}}\right),\left(C_{m_{2,1}, j_{1}}, \ldots, C_{m_{2, r_{2}}, j_{r_{2}}}\right)$ contain an unmixed ramification structure $\left(T_{1}, T_{2}\right)$ of type $\left(\tau_{1}, \tau_{2}\right)$, by Theorem 3.10 (see the proof of Theorem 3.12). 
From Lemma 3.1, since $\mathfrak{S}_{n}=\operatorname{Aut}\left(\mathfrak{A}_{n}\right)$ (for $n>6$ ), we deduce that if $n$ is large enough, then $h\left(\mathfrak{A}_{n} ; \tau_{1}, \tau_{2}\right) \geq k^{r_{1}+r_{2}}$. Now, $k$ can be arbitrarily large, therefore,

$$
h\left(\mathfrak{A}_{n} ; \tau_{1}, \tau_{2}\right) \stackrel{n \rightarrow \infty}{\longrightarrow} \infty .
$$

Moreover, as the number of different almost homogeneous conjugacy classes in $\mathfrak{S}_{n}$ of some certain order grows linearly in $n$, the proof actually shows that $h=\Omega\left(n^{r_{1}+r_{2}}\right)$.

Similarly, we can show that if $\tau_{1}=\left(m_{1,1}, \ldots, m_{1, r_{1}}\right)$ and $\tau_{2}=\left(m_{2,1}, \ldots, m_{2, r_{2}}\right)$ are two sequences of natural numbers such that $m_{i, l} \geq 2$, at least two of $\left(m_{l, 1}, \ldots, m_{l, r_{l}}\right)$ are even and $\sum_{l=1}^{r_{i}}\left(1-1 / m_{i, l}\right)>2$, for $i=1,2$, then

$$
h\left(\mathfrak{S}_{n} ; \tau_{1}, \tau_{2}\right) \stackrel{n \rightarrow \infty}{\longrightarrow} \infty,
$$

and moreover, $h=\Omega\left(n^{r_{1}+r_{2}}\right)$, thus proving Theorem 1.6(a).

3.3. Hurwitz components for $\operatorname{PSL}(2, p)$. In this section we prove Theorem 1.9. The proof is based on well-known properties of the group $\operatorname{PSL}\left(2, p^{e}\right)$ (see for example $[\mathrm{Su}]$ ) and on results of Macbeath [Ma].

Let $q=p^{e}$, where $p$ is an odd prime and $e \geq 1$. Recall that $\operatorname{GL}(2, q)$ is the group of invertible $2 \times 2$ matrices over the finite field with $q$ elements, which we denote by $\mathbb{F}_{q}$, and $\mathrm{SL}(2, q)$ is the subgroup of $\mathrm{GL}(2, q)$ comprising the matrices with determinant 1 . Then $\operatorname{PGL}(2, q)$ and $\operatorname{PSL}(2, q)$ are the quotients of $\operatorname{GL}(2, q)$ and $\operatorname{SL}(2, q)$ by their respective centers. Moreover we can identify $\operatorname{PSL}(2, q)$ with a normal subgroup of index 2 in $\operatorname{PGL}(2, q)$.

Since all non-trivial elements in $\operatorname{PSL}(2, q)$, whose pre-images in $\operatorname{SL}(2, q)$ have the same trace, are conjugate in $\operatorname{PGL}(2, q)$, all of them have the same order in $\operatorname{PSL}(2, q)$. Therefore, we may denote by $\mathcal{O} r d(\alpha)$ the order in $\operatorname{PSL}(2, q)$ of the image of a non-trivial matrix $A \in \mathrm{SL}(2, q)$ whose trace equals $\alpha$, and denote, for an integer $l$,

$$
\mathcal{T}_{l}=\left\{\alpha \in \mathbb{F}_{q}: \mathcal{O r d}(\alpha)=l\right\} .
$$

Note that since $q$ is odd then $\alpha \in \mathcal{T}_{l}$ if and only if $-\alpha \in \mathcal{T}_{l}$.

Now, one can easily compute the size of $\mathcal{T}_{l}$ for any integer $l$.

Lemma 3.15. Let $p$ be an odd prime and let $q=p^{e}$. Then in $\operatorname{PSL}(2, q)$,

(i) $\mathcal{T}_{p}=\{ \pm 2\}$ and so $\left|\mathcal{T}_{p}\right|=2$.

(ii) $\mathcal{T}_{2}=\{0\}$ and so $\left|\mathcal{T}_{2}\right|=1$.

(iii) If $r \geq 3$ and $r \mid \frac{q \pm 1}{2}$ then $\left|\mathcal{T}_{r}\right|=\phi(r)$, where $\phi$ is the Euler function.

(iv) For other values of $r,\left|\mathcal{T}_{r}\right|=0$.

This Lemma is immediate, but for the convenience of the reader we shall present a proof of part (iii).

Proof of Lemma 3.15 (iii). Assume that $r \mid \frac{q-1}{2}$ (if $r \mid \frac{q+1}{2}$ then the proof is similar). Let $\lambda$ be a primitive root of unity of order $2 r$ in $\mathbb{F}_{q}$, then there are $2 \phi(r)$ diagonal matrices whose images in $\operatorname{PSL}(2, q)$ have exact order $r$, parametrized by $\left\{ \pm \lambda^{i}: 1 \leq i \leq 2 r,(i, 2 r)=1\right\}$, if $r$ is odd, or by $\left\{ \pm \lambda^{i}: 1 \leq i \leq r,(i, 2 r)=1\right\}$, if $r$ is even. Hence, there are exactly $\phi(r)$ different traces of diagonal matrices of order $r$, given as $\left\{ \pm \alpha_{1}, \ldots, \pm \alpha_{\psi}\right\}$, where $\psi=\frac{\phi(r)}{2}$. 
In order to estimate the number of Hurwitz components for $\operatorname{PSL}(2, p)$, we would first like to estimate the number $d(\operatorname{PSL}(2, p) ; \tau)$ for certain types $\tau$ (see Corollaries 3.5 and 3.6).

Recall that by Corollary 3.5 ,

$$
d(\operatorname{PSL}(2, p) ; \tau)=\#\left\{O_{T^{u n}}^{\mathrm{PSL}(2, p)}: T \in \mathcal{S}(\operatorname{PSL}(2, p), \tau)\right\} .
$$

Thus, $d(\operatorname{PSL}(2, p) ; \tau)=2 d^{\prime}(\operatorname{PSL}(2, p), \tau)$, where

$$
d^{\prime}(\operatorname{PSL}(2, p) ; \tau):=\#\left\{O_{T^{u n}}^{\mathrm{PGL}(2, p)}: T \in \mathcal{S}(\operatorname{PSL}(2, p), \tau)\right\} .
$$

One can compute $d^{\prime}(\operatorname{PSL}(2, p), \tau)$ using the following Lemma, which follows from results of Macbeath [Ma].

Lemma 3.16. Let $2 \leq l \leq m \leq n$ and assume that $m>2$ and $n>5$. Then

$$
\begin{aligned}
& d^{\prime}(\operatorname{PSL}(2, p) ;(l, m, n))=\#\left\{( \pm \alpha, \pm \beta, \pm \gamma): \alpha \in \mathcal{T}_{l}, \beta \in \mathcal{T}_{m}, \gamma \in \mathcal{T}_{n},\right. \\
& \left.\quad \text { and either } \alpha^{2}+\beta^{2}+\gamma^{2}-\alpha \beta \gamma \neq 4 \text { or } \alpha^{2}+\beta^{2}+\gamma^{2}+\alpha \beta \gamma \neq 4\right\} .
\end{aligned}
$$

Proof. Let $(\alpha, \beta, \gamma) \in \mathbb{F}_{p}^{3}$ such that $\alpha \in \mathcal{T}_{l}, \beta \in \mathcal{T}_{m}$ and $\gamma \in \mathcal{T}_{n}$. In this case, also $-\alpha \in \mathcal{T}_{l},-\beta \in \mathcal{T}_{m}$ and $-\gamma \in \mathcal{T}_{n}$.

Then, by [Ma, Theorem 1], there exist three matrices $A, B, C \in \mathrm{SL}(2, p)$ such that $A B C=1, \operatorname{tr}(A)=\alpha, \operatorname{tr}(B)=\beta$ and $\operatorname{tr}(C)=\gamma$. Denote by $\bar{A}$ the image of a matrix $A$ in $\operatorname{PSL}(2, p)$. Then, $\bar{A}$ has order $l, \bar{B}$ has order $m$ and $\bar{C}$ has order $n$.

By [Ma, Theorem 4], the group generated by $\bar{A}$ and $\bar{B}$ is $\operatorname{PSL}(2, p)$ if and only if either $\alpha^{2}+\beta^{2}+\gamma^{2}-\alpha \beta \gamma \neq 4$ or $\alpha^{2}+\beta^{2}+\gamma^{2}+\alpha \beta \gamma \neq 4$.

In addition, if there exist some other matrices $A^{6}, B^{6}, C^{6} \in \mathrm{SL}(2, p)$ such that $A^{6} B^{6} C^{6}=1, \operatorname{tr}\left(A^{6}\right)= \pm \alpha, \operatorname{tr}\left(B^{6}\right)= \pm \beta$ and $\operatorname{tr}\left(C^{6}\right)= \pm \gamma$ then by [Ma, Theorem 3], there is some $g \in \operatorname{PGL}(2, p)$ such that $g \bar{A} g^{-1}=\bar{A}^{\natural}$ and $g \bar{B} g^{-1}=\bar{B}^{\zeta}$ implying that also $g \bar{C} g^{-1}=g \bar{B}^{-1} \bar{A}^{-1} g^{-1}=\bar{C}^{\zeta}$.

Corollary 3.17. Let $p \geq 5$ be an odd prime, then in $\operatorname{PSL}(2, p)$,

(i) $d^{\prime}(\operatorname{PSL}(2, p) ;(2,3, p))=1$.

(ii) If $r \geq 7$ and $r \mid \frac{p \pm 1}{2}$ then $d^{\prime}(\operatorname{PSL}(2, p) ;(2,3, r))=\frac{\phi(r)}{2}$.

(iii) $d^{\prime}(\operatorname{PSL}(2, p) ;(p, p, p))=1$.

(iv) If $r \geq 7$ and $r \mid \frac{p \pm 1}{2}$ then

$$
d^{\prime}(\operatorname{PSL}(2, p) ;(r, r, r))=\frac{\psi(\psi+1)(\psi+2)}{6},
$$

where $\psi=\frac{\phi(r)}{2}$.

(v) If $2<l<m<n$ such that $n>5$ and $l, m, n$ all divide $\frac{p \pm 1}{2}$, then

$$
d^{\prime}(\operatorname{PSL}(2, p) ;(l, m, n))=\frac{\phi(l) \phi(m) \phi(n)}{8} .
$$

(vi) If $2 \leq l \leq m \leq n$ such that $m>2$ and $n>5$ then

$$
d^{\prime}(\operatorname{PSL}(2, p) ;(l, m, n)) \leq \frac{\phi(l) \phi(m) \phi(n)}{8} .
$$

Proof. The proof is based on Lemma 3.15 and Lemma 3.16

(i) The orders $(2,3, p)$ correspond to the traces $(0, \pm 1, \pm 2)$. 
(ii) The orders $(2,3, r)$ correspond to the traces $(0, \pm 1, \pm \gamma)$, with $\mathcal{O r d}(\gamma)=$ $r$. We need to verify that $0^{2}+1^{2}+\gamma^{2}-0 \neq 4$. Indeed, $0^{2}+1^{2}+\gamma^{2}-0=$ 4 is equivalent to $\gamma^{2}=3$, and $\gamma^{2}=3$ if and only if $\mathcal{O r d}(\gamma)=6$, a contradiction.

(iii) The orders $(p, p, p)$ correspond to the traces $(-2,-2,2)$ (see $\mathrm{Ma}$, Theorem 7]).

(iv) The orders $(r, r, r)$ correspond to the traces $\left( \pm \alpha_{i}, \pm \alpha_{j}, \pm \alpha_{k}\right)$ for $1 \leq$ $i \leq j \leq k \leq \psi$. If $\alpha_{i}^{2}+\alpha_{j}^{2}+\alpha_{k}^{2}-\alpha_{i} \alpha_{j} \alpha_{k}=4$, then $\alpha_{i}^{2}+\alpha_{j}^{2}+$ $\alpha_{k}^{2}-\alpha_{i} \alpha_{j} \alpha_{k} \neq 4$, hence, if necessary, we may replace $\left(\alpha_{i}, \alpha_{j}, \alpha_{k}\right)$ by $\left(-\alpha_{i},-\alpha_{j},-\alpha_{k}\right)$. Therefore,

$$
d^{\prime}(\operatorname{PSL}(2, p) ;(r, r, r))=\left(\begin{array}{l}
\psi \\
3
\end{array}\right)+2\left(\begin{array}{l}
\psi \\
2
\end{array}\right)+\psi=\frac{\psi(\psi+1)(\psi+2)}{6} .
$$

(v) The orders $(l, m, n)$ correspond to the traces $(\alpha, \beta, \gamma)$ where $\mathcal{O} r d(\alpha)=$ $l, \mathcal{O} r d(\beta)=m, \mathcal{O} r d(\gamma)=n$, and $\alpha, \beta, \gamma \neq 0$. Now, we may replace $(\alpha, \beta, \gamma)$ by $(-\alpha,-\beta,-\gamma)$, if necessary.

(vi) This follows from the previous calculations.

Proof of Theorem 1.9. Let $p$ be an odd prime, and let $\tau_{1}=\left(r_{1}, s_{1}, t_{1}\right)$ and $\tau_{2}=\left(r_{2}, s_{2}, t_{2}\right)$ be two hyperbolic types. By Corollary 3.17, for $i=1,2$, $d^{\prime}\left(\operatorname{PSL}(2, p) ;\left(r_{i}, s_{i}, t_{i}\right)\right)$ is maximal when $r_{i}, s_{i}$ and $t_{i}$ are three different integers dividing $\frac{p \pm 1}{2}$, and hence is at most $\frac{\phi\left(r_{i}\right) \phi\left(s_{i}\right) \phi\left(t_{i}\right)}{8}$.

Define the following constant

$$
c:=\frac{\phi\left(r_{1}\right) \phi\left(s_{1}\right) \phi\left(t_{1}\right) \phi\left(r_{2}\right) \phi\left(s_{2}\right) \phi\left(t_{2}\right)}{16} .
$$

Then, by Corollary 3.6,

$$
h\left(G ; \tau_{1}, \tau_{2}\right) \leq d\left(G ; \tau_{1}\right) \cdot d\left(G ; \tau_{2}\right)=2 d^{\prime}\left(G ; \tau_{1}\right) \cdot 2 d^{\prime}\left(G ; \tau_{2}\right) \leq c .
$$

\subsection{Ramification structures and Hurwitz components for abelian} groups and their extensions. In this Section we generalize previous results regarding abelian groups and their extensions, which appeared in [BCG05.

3.4.1. Ramification structures of abelian groups. The following Theorem generalizes [BCG05, Theorem 3.4] in case $G$ abelian and $S$ is isogeneous to a higher product (not necessarily Beauville).

From now on we use the additive notation for abelian groups.

Theorem 3.18. Let $G$ be an abelian group, given as

$$
G \cong \mathbb{Z} / n_{1} \mathbb{Z} \times \cdots \times \mathbb{Z} / n_{t} \mathbb{Z},
$$

where $n_{1}|\cdots| n_{t}$. For a prime $p$, denote by $l_{i}(p)$ the largest power of $p$ which divides $n_{i}$ (for $\left.1 \leq i \leq t\right)$.

Let $r_{1}, r_{2} \geq 3$, then $G$ admits an unmixed ramification structure of size $\left(r_{1}, r_{2}\right)$ if and only if the following conditions hold:

- $r_{1}, r_{2} \geq t+1$

- $n_{t}=n_{t-1}$;

- If $l_{t-1}(3)>l_{t-2}(3)$ then $r_{1}, r_{2} \geq 4$; 
- $l_{t-1}(2)=l_{t-2}(2)$;

- If $l_{t-2}(2)>l_{t-3}(2)$ then $r_{1}, r_{2} \geq 5$ and $r_{1}, r_{2}$ are not both odd.

Proof. Let $\left(x_{1}, \ldots, x_{r_{1}} ; y_{1}, \ldots, y_{r_{2}}\right)$ be an unmixed ramification structure of size $\left(r_{1}, r_{2}\right)$. Set

$$
\Sigma_{1}:=\Sigma\left(x_{1}, \ldots, x_{r_{1}}\right):=\left\{i_{1} x_{1}, \ldots, i_{r_{1}} x_{r_{1}}: i_{1}, \ldots i_{r_{1}} \in \mathbb{Z}\right\},
$$

and

$$
\Sigma_{2}:=\Sigma\left(y_{1}, \ldots, y_{r_{2}}\right):=\left\{j_{1} y_{1}, \ldots, j_{r_{2}} y_{r_{2}}: j_{1}, \ldots j_{r_{2}} \in \mathbb{Z}\right\},
$$

and recall that $\Sigma_{1} \cap \Sigma_{2}=\{0\}$.

Consider the primary decomposition of $G$,

$$
G=\bigoplus_{p \in\{\text { Primes }\}} G_{p}
$$

and observe that since $G$ is generated by $\min \left\{r_{1}, r_{2}\right\}-1$ elements, so is any $G_{p}$ (which is a characteristic subgroup of $G$ ).

Therefore, $G_{p}$ can be written as

$$
G_{p} \cong \mathbb{Z} / p^{k_{1}} \mathbb{Z} \times \cdots \times \mathbb{Z} / p^{k_{t-1}} \mathbb{Z} \times \mathbb{Z} / p^{k_{t}} \mathbb{Z},
$$

where $k_{1} \leq \cdots \leq k_{t-1} \leq k_{t}$ and $1 \leq t \leq \min \left\{r_{1}, r_{2}\right\}-1$.

Denote $H_{p}:=p^{k_{t}-1} G_{p}$, and observe that $H_{p}$ is an elementary abelian group of rank at most $t$.

Step 1. Let $x_{1}=\left(x_{1, p}\right) \in \bigoplus_{p \in\{\text { Primes }\}} G_{p}$ and let

$$
\Sigma_{1, p}:=\Sigma\left(x_{1, p}, \ldots, x_{r_{1}, p}\right):=\left\{l_{1} x_{1, p}, \ldots, l_{r_{1}} x_{r_{1}, p}: l_{1}, \ldots l_{r_{1}} \in \mathbb{Z}\right\},
$$

be the set of multiples of $\left(x_{1, p}, \ldots, x_{r_{1}, p}\right)$, then by the Chinese Remainder Theorem, $x_{1, p}$ is a multiple of $x_{1}$, and hence $\Sigma_{1} \supseteq \Sigma_{1, p}$.

Step 2. $G_{p}$ is not cyclic.

Otherwise, if $G_{p} \cong \mathbb{Z} / p^{k} \mathbb{Z}$, then $H_{p}=p^{k-1} G_{p} \cong \mathbb{Z} / p \mathbb{Z}$. Since $\Sigma_{1, p}$ contains a generator of $G_{p}$, it also contains a non-trivial element of $H_{p}$ and so $\Sigma_{1, p} \supseteq H_{p}$. Thus $\Sigma_{1} \supseteq H_{p}$, and similarly $\Sigma_{2} \supseteq H_{p}$, a contradiction to $\Sigma_{1} \cap \Sigma_{2}=\{0\}$.

Step 3. $k_{t}=k_{t-1}$, namely $G_{p} \cong \mathbb{Z} / p^{k_{1}} \mathbb{Z} \times \cdots \times \mathbb{Z} / p^{k_{t-1}} \mathbb{Z} \times \mathbb{Z} / p^{k_{t-1}} \mathbb{Z}$, where $k_{1} \leq \cdots \leq k_{t-1}$ and $2 \leq t \leq \min \left\{r_{1}, r_{2}\right\}-1$.

Otherwise, if $k_{t} \neq k_{t-1}$, then $H_{p}=p^{k_{t}-1} G_{p} \cong \mathbb{Z} / p \mathbb{Z}$. As in Step $2, \Sigma_{1, p}$ contains a generator of $G_{p}$, and so it also contains a non-trivial element of $H_{p}$. Thus $\Sigma_{1, p} \supseteq H_{p}$, and similarly $\Sigma_{2, p} \supseteq H_{p}$, a contradiction to $\Sigma_{1} \cap \Sigma_{2}=\{0\}$.

Step 4. $p=2$ or 3 .

The extra conditions for $p=2$ and 3 are due to dimensional reasons.

- Let $p=2$ and assume that $k_{t-1}>k_{t-2}$. In this case, $H_{2} \cong(\mathbb{Z} / 2 \mathbb{Z})^{2}$ contains only three non-trivial vectors. However, $\left|H_{2} \cap \Sigma_{1,2}\right| \geq 2$ and $\left|H_{2} \cap \Sigma_{2,2}\right| \geq 2$, a contradiction to $\Sigma_{1} \cap \Sigma_{2}=\{0\}$.

- Let $p=2$ and assume that $k_{t-1}=k_{t-2}>k_{t-3}$. In this case, $H_{2} \cong$ $(\mathbb{Z} / 2 \mathbb{Z})^{3}$ contains only seven non-trivial vectors.

If $r_{1}=4$ then $\Sigma_{1,2}$ contains four different vectors which generate $H_{2}$, whose sum is zero, say $\left\{e_{1}, e_{2}, e_{3}, e_{1}+e_{2}+e_{3}\right\}$. Now, the other three vectors in $H_{2}$ are necessarily $\left\{e_{1}+e_{2}, e_{1}+e_{3}, e_{2}+e_{3}\right\}$, which are linearly dependent, and so cannot generate $H_{2} \cong(\mathbb{Z} / 2 \mathbb{Z})^{3}$. 
When $r_{1}$ is odd, $\Sigma_{1,2}$ contains four different vectors from $H_{2}$. Indeed, a sum $x_{1}+\cdots+x_{r_{1}}$ of some vectors $v, u, w$ over $\mathbb{Z} / 2 \mathbb{Z}$ (i.e. $x_{i} \in\{v, u, w\}$ ), where $r_{1}$ is odd, cannot be equal to 0 , unless $v, u$ and $w$ are linearly dependent, and so cannot generate $H_{2} \cong(\mathbb{Z} / 2 \mathbb{Z})^{3}$. Thus, if $r_{1}$ is odd, then $\left|H_{2} \cap \Sigma_{1,2}\right| \geq 4$, and similarly, if $r_{2}$ is odd, then $\left|H_{2} \cap \Sigma_{2,2}\right| \geq 4$, a contradiction to $\Sigma_{1} \cap \Sigma_{2}=\{0\}$.

- Let $p=3$ and assume that $k_{t-1}>k_{t-2}$. In this case, $H_{3} \cong(\mathbb{Z} / 3 \mathbb{Z})^{2}$ contains only eight non-trivial vectors. If $r_{1}=3$ then $\Sigma_{1,3}$ contains three different vectors, which generate $H_{3}$, whose sum is zero, say $\left\{e_{1}, e_{2}, 2 e_{1}+2 e_{2}\right\}$, as well as their multiples $\left\{2 e_{1}, 2 e_{2}, e_{1}+e_{2}\right\}$. Now, the other two vectors in $H_{2}$ are necessarily $\left\{e_{1}+2 e_{2}, 2 e_{1}+e_{2}\right\}$, which are linearly dependent, and so cannot generate $H_{3} \cong(\mathbb{Z} / 3 \mathbb{Z})^{2}$.

Step 5. Now, let $p \geq 5$ and assume that $G_{p}=\mathbb{Z} / p^{k_{1}} \mathbb{Z} \times \cdots \times \mathbb{Z} / p^{k_{t-1}} \mathbb{Z} \times$ $\mathbb{Z} / p^{k_{t-1}} \mathbb{Z}$, where $k_{1} \leq \cdots \leq k_{t-1}$ and $2 \leq t \leq \min \left\{r_{1}, r_{2}\right\}-1$. We will choose appropriate vectors for $\Sigma_{1, p}$ and $\Sigma_{2, p}$.

Assume that $(a, b, c, d)$ satisfy the condition in Equation (11) below, and let

$$
\begin{array}{rlrl}
x_{1, p} & =(1,0, \ldots, 0,1,0) & y_{1, p} & =(1,0, \ldots, 0, a, b) \\
x_{2, p} & =(0,1,0, \ldots, 0,0,1) & y_{2, p} & =(0,1,0, \ldots, 0, c, d) \\
x_{3, p} & =(0,0,1,0, \ldots, 0,-1,0) & y_{3, p} & =(0,0,1,0, \ldots, 0,-a,-b) \\
x_{4, p} & =(0,0,0,1,0 \ldots, 0,0,-1) & y_{4, p} & =(0,0,0,1,0, \ldots, 0,-c,-d) \\
\vdots & \vdots & \\
x_{t-2, p} & =(0, \ldots, 0,1, *, *) & y_{t-2, p} & =(0, \ldots, 0,1, *, *) \\
x_{t-1, p} & =(0, \ldots, 0,0, *, *) & y_{t-1, p} & =(0, \ldots, 0,0, *, *) \\
x_{t, p} & =(0, \ldots, 0,0, *, *) & y_{t, p} & =(0, \ldots, 0,0, *, *) \\
\vdots & & \vdots & \\
x_{r_{1}, p} & =(-1, \ldots,-1,-1,-1) & y_{r_{2}, p} & =(-1, \ldots,-1,-a-c,-b-d)
\end{array}
$$

where the elements marked with $(*, *)$ in $x_{t-2, p}$ (and after) are chosen from $\{(0, \pm 1),( \pm 1,0), \pm(1,1)\}$ such that $\left(x_{1, p}, x_{2, p}, \ldots, x_{t, p}\right)$ are independent and the sum $x_{1, p}+\cdots+x_{r_{1}, p}=0$. Similarly, the elements marked with $(*, *)$ in $y_{t-2, p}$ (and after) are chosen from $\{ \pm(a, b), \pm(c, d), \pm(a+c, b+d)\}$, such that $\left(y_{1, p}, y_{2, p}, \ldots, y_{t, p}\right)$ are independent and $y_{1, p}+\cdots+y_{r_{1}, p}=0$.

Since $\left\langle x_{1, p}, \ldots, x_{r_{1}, p}\right\rangle=G_{p}=\left\langle y_{1, p}, \ldots, y_{r_{2}, p}\right\rangle$, we deduce that $\left(x_{1, p}, \ldots, x_{r_{1}, p}\right)$ form a spherical $r_{1}$-system of generators for $G_{p}$ and that $\left(y_{1, p}, \ldots, y_{r_{2}, p}\right)$ form a spherical $r_{2}$-system of generators for $G_{p}$. Moreover, for every $1 \leq i \leq r_{1}, 1 \leq j \leq r_{2}$, and $k, l \in \mathbb{Z}$, if the vectors $k x_{i, p}$ and $l y_{j, p}$ are not trivial, then they are linearity independent. Hence, $\Sigma_{1, p} \cap \Sigma_{2, p}=\{0\}$, as needed.

When $p=2$ or 3 it suffices to construct unmixed ramification structures for the elementary abelian groups in characteristic 2 and 3 . These yield an unmixed ramification structure for any choice of $H_{2}$ (resp. $H_{3}$ ), which induces an appropriate structure for any $G_{2}$ (resp. $G_{3}$ ), by completing the 
generating vectors of $H_{2}$ (resp. $\left.H_{3}\right)$ to generating vectors of $G_{2}$ (resp. $G_{3}$ ), essentially in the same way of $p \geq 5$. These constructions are described in the following Lemmas 3.19 and 3.20 .

Now, recall that by using the primary decomposition of $G$, it was enough to check the conditions on each primary component $G_{p}$, thus $G$ admits an unmixed ramification structure of size $\left(r_{1}, r_{2}\right)$ as needed.

Lemma 3.19. Let $G=(\mathbb{Z} / 2 \mathbb{Z})^{t}$.

If $t \geq 4$ then $G$ always admits an unmixed ramification structure of size $\left(r_{1}, r_{2}\right)$, for any $r_{1}, r_{2} \geq t+1$.

If $t=3$ then $G$ admits an unmixed ramification structure of size $\left(r_{1}, r_{2}\right)$, if and only if $r_{1}, r_{2} \geq 5$ and $r_{1}, r_{2}$ are not both odd.

Lemma 3.20. Let $G=(\mathbb{Z} / 3 \mathbb{Z})^{t}$.

If $t \geq 3$ then $G$ always admits an unmixed ramification structure of size $\left(r_{1}, r_{2}\right)$, for any $r_{1}, r_{2} \geq t+1$.

If $t=2$ then $G$ admits an unmixed ramification structure of size $\left(r_{1}, r_{2}\right)$, if and only if $r_{1}, r_{2} \geq 4$.

The proofs of both lemmas are straight forward calculation.

Lemma 3.21. Let $p \geq 5$ be a prime number and $U:=(\mathbb{Z} / p \mathbb{Z})^{*}$, the number $N$ of quadruples $(a, b, c, d) \in U$ such that:

$$
a-b, a+c, c-d, b+d, a+c-b-d, a d-b c \in U
$$

is $N=(p-1)(p-2)(p-3)(p-4)$.

Proof. The number $N$ equals $p-1$ times the number of solutions that we get for $a=1$. Now, $b \neq 0,1$, so there are $p-2$ possibilities for $b$. The conditions $c \neq 0,-1$ and $d \neq 0,-b$ imply $(p-2)^{2}$ possibilities for the pair $(c, d)$. From this number we need to subtract the number of solutions for $c=d, d=1-b+c$ and $d=b c$, which are $p-2, p-2$ and $p-4$ respectively. We deduce that there are $(p-2)^{2}-[(p-2)+(p-2)+(p-4)]=(p-3)(p-4)$ possibilities for the pair $(c, d)$. Hence $N=(p-1)(p-2)(p-3)(p-4)$.

We remark that this Lemma corrects the calculation given in BCG05, Theorem 3.4].

3.4.2. Hurwitz components in case $(\mathbb{Z} / n \mathbb{Z})^{2}$. Observe that for $G=(\mathbb{Z} / n \mathbb{Z})^{2}$ there is only one type of a spherical 3-system of generators, which is $\tau=$ $(n, n, n)$. Also note that $\operatorname{Aut}(G) \cong \mathrm{GL}(2, n)$.

The following Lemmas give a more precise estimation of the number of Hurwitz components in case $G=(\mathbb{Z} / n \mathbb{Z})^{2}$, which generalizes Remark 3.5 in BCG05.

Lemma 3.22. Let $p \geq 5$ be a prime. The number $h=h(G ; \tau, \tau)$, where $\tau=(p, p, p)$, of Hurwitz components for $G=(\mathbb{Z} / p \mathbb{Z})^{2}$ satisfies

$$
N_{p} / 72 \leq h \leq N_{p} / 6,
$$

where $N_{p}=(p-1)(p-2)(p-3)(p-4)$.

Proof. Let $\left(x_{1}, x_{2} ; y_{1}, y_{2}\right)$ be an unmixed Beauville structure for $G$. Since $x_{1}, x_{2}$ are generators of $G$, they are a basis, and without loss of generality $x_{1}, x_{2}$ are the standard basis $x_{1}=(1,0), x_{2}=(0,1)$. Now, let $y_{1}=(a, b)$, 
$y_{2}=(c, d)$, then the condition $\Sigma_{1} \cap \Sigma_{2}=\{0\}$ means that any pair of the six vectors yield a basis of $G$, implying that $a, b, c, d$ must satisfy the conditions given in Equation (11).

Moreover, the $N_{p}$ pairs $((1,0),(0,1) ;(a, b),(c, d))$, where $a, b, c, d$ satisfy (11), are exactly the representatives for the $\operatorname{Aut}(G)$-orbits in the set $\mathcal{U}(G ; \tau, \tau)$.

Now, one should consider the action of $B_{3} \times B_{3}$ on $\mathcal{U}(G ; \tau, \tau)$, which is equivalent to the action of $\mathfrak{S}_{3} \times \mathfrak{S}_{3}$, since $G$ is abelian. The action of $\mathfrak{S}_{3}$ on the second component is obvious (there are 6 permutations), and the action of $\mathfrak{S}_{3}$ on the first component can be translated to an equivalent Aut $(G)$-action, given by multiplication in one of the six matrices:

$$
\left(\begin{array}{ll}
1 & 0 \\
0 & 1
\end{array}\right),\left(\begin{array}{ll}
0 & 1 \\
1 & 0
\end{array}\right),\left(\begin{array}{ll}
-1 & 0 \\
-1 & 1
\end{array}\right),\left(\begin{array}{ll}
1 & -1 \\
0 & -1
\end{array}\right),\left(\begin{array}{ll}
-1 & 1 \\
-1 & 0
\end{array}\right),\left(\begin{array}{ll}
0 & -1 \\
1 & -1
\end{array}\right),
$$

yielding an equivalent representative.

Therefore, the action of $\mathfrak{S}_{3}$ on the second component yields orbits of length 6 , and the action of $\mathfrak{S}_{3}$ on the first component connects them together, and gives orbits of sizes from 6 to 36 . Moreover since one can exchange the vector $\left(x_{1}, x_{2}\right)$ with the vector $\left(y_{1}, y_{2}\right)$ we get the desired result.

Corollary 3.23. Let $p \geq 5$ be a prime. The number $h=h(G ; \tau, \tau)$, where $\tau=\left(p^{k}, p^{k}, p^{k}\right)$, of Hurwitz components for $G=\left(\mathbb{Z} / p^{k} \mathbb{Z}\right)^{2}$ satisfies

$$
N_{p^{k}} / 72 \leq h \leq N_{p^{k}} / 6,
$$

where $N_{p^{k}}=p^{4 k-4}(p-1)(p-2)(p-3)(p-4)$.

Proof. In this case, the number $N_{p^{k}}$ of $\operatorname{Aut}(G)$-orbits in the set $\mathcal{U}(G ; \tau, \tau)$ is exactly $p^{4 k-4}$ times $N_{p}$, and the proof is the same as in the previous Lemma 3.22 .

Corollary 3.24. Let $n$ be an integer s.t. $(n, 6)=1$. The number $h=$ $h(G ; \tau, \tau)$, where $\tau=(n, n, n)$, of Hurwitz components for $G=(\mathbb{Z} / n \mathbb{Z})^{2}$, where $n=p_{1}^{k_{1}} \cdot \ldots \cdot p_{t}^{k_{t}}$, satisfies

$$
N_{n} / 72 \leq h \leq N_{n} / 6
$$

where $N_{n}=\prod_{i=1}^{t} p_{i}^{4 k_{i}-4}\left(p_{i}-1\right)\left(p_{i}-2\right)\left(p_{i}-3\right)\left(p_{i}-4\right)$.

Proof. By the Chinese Remainder Theorem, the number $N_{n}$ of Aut $(G)$-orbits in the set $\mathcal{U}(G ; \tau, \tau)$ can be computed using Corollary 3.23, and the proof is now the same as in Lemma 3.22 .

Remark 3.25. It clearly follows that $N_{n}=O\left(n^{4}\right)$. In addition, in [GJT] is given an explicit formula for $N_{n}$.

Notice that if $n$ is divisible by the first $l$ primes $p_{i} \geq 5$ then since:

$$
\lim _{l \rightarrow \infty} \prod_{i}\left(1-\frac{1}{p_{i}}\right)=0
$$

we have $N_{n} / n^{4} \rightarrow 0$ as $l \rightarrow \infty$. 
3.4.3. Hurwitz components in case $G=(\mathbb{Z} / p \mathbb{Z})^{r}$. Fix an integer $r$, let $p>5$ be a prime number, and let $G=(\mathbb{Z} / p \mathbb{Z})^{r}$, then by Theorem 3.18 , $G$ admits an unmixed ramification structure of type $\left(\tau_{1}, \tau_{2}\right)$ where $\tau_{1}=\tau_{2}=\tau=$ $(p, \ldots, p)(p$ appears $(r+1)$-times $)$ and $r_{1}=r_{2}=r+1$.

Proposition 3.26. Fix an integer $r \geq 2$, then the number $h=h(G ; \tau, \tau)$ of Hurwitz components for $G=(\mathbb{Z} / p \mathbb{Z})^{r}$ and $\tau=(p, \ldots, p)$ ( $p$ appears $(r+1)$-times) satisfies, as $p \rightarrow \infty$,

$$
h=\Theta\left(p^{r^{2}}\right) .
$$

Proof. Let $\left(x_{1}, \ldots, x_{r+1} ; y_{1}, \ldots, y_{r+1}\right)$ be an unmixed ramification structure for $G$. Since $x_{1}, \ldots, x_{r+1}$ generate $G$, they are a basis, and without loss of generality they are of the form given in Step 5 of Theorem 3.18. However, for $y_{1}, \ldots, y_{r+1}$ one can take any appropriate set of $r+1$ vectors in $(\mathbb{Z} / p \mathbb{Z})^{r}$, which admit an unmixed ramification structure, and so each proper choice of $\left(y_{1}, \ldots, y_{r+1}\right)$ corresponds to exactly one $\operatorname{Aut}(G)$-orbit in the set $\mathcal{U}(G ; \tau, \tau)$.

Therefore, one can choose any invertible $(r-2) \times(r-2)$ matrix for

$$
\left(\begin{array}{ccc}
y_{1,1} & \cdots & y_{1, r-2} \\
& \vdots & \\
y_{r-2,1} & \cdots & y_{r-2, r-2}
\end{array}\right),
$$

choose any vector of length $r-2$ for $\left(y_{r-1,1}, \ldots, y_{r-1, r-2}\right)$, and similarly for $\left(y_{r, 1}, \ldots, y_{r, r-2}\right)$. Moreover, for $1 \leq i \leq r-2$, one can choose for $\left(y_{i, r-1}, y_{i, r}\right)$ any vector from the set $S:=\left\{(a, b) \in \mathbb{F}_{p}^{2}: a \neq 0, b \neq 0, a \neq b\right\}$. Observe that $|S|=(p-1)(p-2)$.

Now, one has to make sure that $y_{r-1}$ is not a linear combination of $y_{1}, \ldots, y_{r-2}$, by choosing $\left(y_{r-1, r-1}, y_{r-1, r}\right)$ appropriately from $S$, and so there are at least $(p-1)(p-2)-1=p^{2}-3 p+1$ possibilities for this pair. Moreover, one should choose $\left(y_{r, r-1}, y_{r, r}\right)$ appropriately from $S$, such that $y_{r}$ is not some linear combination of $y_{1}, \ldots, y_{r-1}$, and that $\left(y_{r+1, r-1}, y_{r+1, r}\right) \in$ $S$, and so the number of possibilities to the pair $\left(y_{r, r-1}, y_{r, r}\right)$ is at least $(p-3)(p-5)=p^{2}-8 p+15$.

The condition that the pairs $\left(y_{i, r-1}, y_{i, r}\right) \in S$ for $1 \leq i \leq r+1$ is needed to guarantee that for any $k, l \in \mathbb{Z}$ and $1 \leq i, j \leq r+1$, if the vectors $k x_{i}$ and $l y_{j}$ are not trivial, then they are linearity independent, and so $\Sigma_{1} \cap \Sigma_{2}=\{0\}$, as needed.

Hence, the number of $\operatorname{Aut}(G)$-orbits in the $\operatorname{set} \mathcal{U}(G ; \tau, \tau)$ is bounded from below by

$$
\begin{aligned}
& |\operatorname{GL}((r-2), p)| p^{2(r-2)}((p-1)(p-2))^{r-2}\left(p^{2}-3 p+1\right)\left(p^{2}-8 p+15\right) \\
& =\Theta\left(p^{(r-2)^{2}+2(r-2)+2(r-2)+2+2}\right)=\Theta\left(p^{r^{2}}\right) .
\end{aligned}
$$

It is clear that the number of orbits is bounded from above by

$$
|\mathrm{GL}(r, p)|=\Theta\left(p^{r^{2}}\right) .
$$

Now, the action of $B_{r+1} \times B_{r+1}$ on the $\operatorname{Aut}(G)$-orbits of $\mathcal{U}(G ; \tau, \tau)$, is equivalent to the action of $\mathfrak{S}_{r+1} \times \mathfrak{S}_{r+1}$, since $G$ is abelian, and so yields orbits of sizes between $(r+1)$ ! and $((r+1) !)^{2}$. This has no effect on the above asymptotic, however, since $r$ is fixed. 


\subsubsection{Extensions of abelian groups, dihedral groups and small groups.}

Group theoretical proof to Lemma 2.4. If $r_{1}=r_{2}=3$, then the result follows from [BCG05, Proposition 3.2]. Note that when $r_{i}=3$ (for $i=1$ or 2) the above inequality is equivalent to the condition that $\mu_{i}<1$.

If $\mu>1$ then the possible unordered types are

$$
(2,2, n)(n \in \mathbb{N}), \quad(2,3,3), \quad(2,3,4), \quad(2,3,5) .
$$

In the first case, $G \cong D_{n}$ is a dihedral group of order $2 n$, and thus cannot admit an unmixed Beauville structure by [BCG05, Lemma 3.7]. Moreover, $G$ cannot admit an unmixed ramification structure $\left(T_{1}, T_{2}\right)$, where $T_{1}$ has an unordered type $(2,2, n)$. Indeed, let $C_{n}$ denote a maximal cyclic subgroup of $D_{n}$, then $D_{n} \backslash C_{n}$ contains at most two conjugacy classes, more precisely, it contains one if $n$ is odd and two if $n$ is even. If $n$ is odd, since both $T_{1}$ and $T_{2}$ contain elements of $D_{n} \backslash C_{n}$, then $\Sigma_{1} \cap \Sigma_{2} \neq\{1\}$. If $n$ is even, then $T_{1}$ necessarily contains two elements from two different conjugacy classes of $D_{n} \backslash C_{n}$, and $T_{2}$ always contains an element of $D_{n} \backslash C_{n}$, which again contradicts $\Sigma_{1} \cap \Sigma_{2}=\{1\}$.

In the other cases, one obtains the following isomorphisms of triangular groups

$$
\Delta(2,3,3) \cong \mathfrak{A}_{4}, \quad \Delta(2,3,4) \cong \mathfrak{S}_{4}, \quad \Delta(2,3,5) \cong \mathfrak{A}_{5},
$$

and it is easy to check that these groups do not admit an unmixed Beauville structure (see also BCG05, Proposition 3.6]). Moreover, these groups cannot admit an unmixed ramification structure $\left(T_{1}, T_{2}\right)$, where $T_{1}$ has an unordered type $(2,3, n)$ and $n=3,4,5$. Indeed, in the groups $\mathfrak{A}_{4}$ and $\mathfrak{A}_{5}$, any two elements of the same order are either conjugate or one can be conjugated to some power of the other, in contradiction to $\Sigma_{1} \cap \Sigma_{2}=\{1\}$. For the group $\mathfrak{S}_{4}, T_{1}$ necessarily contains one 2-cycle, one 3-cycle and one 4-cycle, so the condition $\Sigma_{1} \cap \Sigma_{2}=\{1\}$ implies that $T_{2}$ can contain only elements which have exactly two 2-cycles, and these elements cannot generate $\mathfrak{S}_{4}$, yielding a contradiction.

If $\mu=1$ then the possible unordered types are

$$
(3,3,3), \quad(2,4,4), \quad(2,3,6),
$$

and so $G$ is a finite quotient of one of the wall-paper groups and cannot admit an unmixed Beauville structure by BCG05, §6]. Moreover, the arguments in BCG05, §6] show that, in fact, these groups cannot admit an unmixed ramification structure $\left(T_{1}, T_{2}\right)$, where $T_{1}$ has an unordered type either $(3,3,3)$ or $(2,4,4)$ or $(2,3,6)$. For example, if $G$ is a quotient of the triangle group $\Delta(3,3,3)$, and we denote by $A$ the maximal normal abelian subgroup of $G$, then by [BCG05, Proposition 6.3], for any $g \in G \backslash A$ there exists some integer $i$ s.t. $g^{i}$ belongs to one of two fixed conjugacy classes $C_{1}$ and $C_{2}$. Moreover, $T_{1}$ necessarily contains two elements $g_{1}, g_{2} \in G \backslash A$ such that $g_{1}^{i_{1}} \in C_{1}$ and $g_{2}^{i_{2}} \in C_{2}$ for some $i_{1}, i_{2}$. Since $T_{2}$ always contains an element of $G \backslash A$, this contradicts $\Sigma_{1} \cap \Sigma_{2}=\{1\}$. 
For $r_{1}, r_{2} \geq 4$ the above inequality holds, unless the type is $(2,2,2,2)$. In the latter case, $G$ is a finite quotient of the wall-paper group

$$
\begin{aligned}
\Gamma & \cong\left\langle t_{1}, t_{2}, t_{3}, t_{4}: t_{1}^{2}, t_{2}^{2}, t_{3}^{2}, t_{4}^{2}, t_{1} t_{2} t_{3} t_{4}\right\rangle \cong\left\langle t_{1}, t_{2}, t_{3}: t_{1}^{2}, t_{2}^{2}, t_{3}^{2},\left(t_{1} t_{2} t_{3}\right)^{2}\right\rangle \\
& \cong\left\langle t, r, s:[r, s], t^{2}, t r t r, t s t s\right\rangle,
\end{aligned}
$$

by setting $t_{1}=r t, t_{2}=t s$ and $t_{3}=t$.

Hence, $\Gamma \cong \mathbb{Z} / 2 \mathbb{Z} \ltimes \mathbb{Z}^{2}$, and so all its finite quotients are of the form $G=$ $\mathbb{Z} / 2 \mathbb{Z} \ltimes(\mathbb{Z} / m \mathbb{Z} \times \mathbb{Z} / n \mathbb{Z})$ for some $m, n \in \mathbb{N}$. We will show in Proposition 3.27 that these groups cannot admit an unmixed ramification structure of size $(4,4)$. In fact, the same argument also shows that these groups cannot admit an unmixed ramification structure $\left(T_{1}, T_{2}\right)$, where $T_{1}$ has an unordered type $(2,2,2,2)$ (see Remark 3.28).

The above proof uses the following proposition, which generalizes the result in [BCG05, Lemma 3.7], that dihedral groups do not admit unmixed Beauville structures.

Proposition 3.27. For any $n, m \in \mathbb{N}$, the finite group

$$
G=\mathbb{Z} / 2 \mathbb{Z} \ltimes(\mathbb{Z} / m \mathbb{Z} \times \mathbb{Z} / n \mathbb{Z})
$$

does not admit an unmixed ramification structure of size $(4,4)$.

Proof. Observe that $G$ can be presented by

$$
G=\left\langle t, r, s: t^{2}, r^{m}, s^{n},[r, s], t s t s, t r t r\right\rangle,
$$

and so any element in $G$ can be written uniquely as $t^{\epsilon} r^{i} s^{j}$ for $\epsilon \in\{0,1\}$, $1 \leq i \leq m, 1 \leq j \leq n$.

Conjugation of some element $t r^{i} s^{j}$ by $r^{-1}$ yields $r^{-1} t r^{i} s^{j} r=t t r^{-1} t r^{i} s^{j} r=$ $t r r^{i} r s^{j}=t r^{i+2} s^{j}$, and similarly conjugation by $s^{-1}$ yields $t r^{i} s^{j+2}$. Hence, $t r^{i} s^{j}$ can be conjugated to $t r^{i+2 k} s^{j+2 l}$ for any $k, l$.

Let $A \cong \mathbb{Z} / m \mathbb{Z} \times \mathbb{Z} / n \mathbb{Z}$ be the maximal normal abelian subgroup in $G$, then $G \backslash A$ contains at most four conjugacy classes, represented by $t, t r, t s, t r s$. In fact, it contains one conjugacy class if both $m, n$ are odd, two conjugacy classes if one of $m, n$ is odd and the other is even, and four if both $m, n$ are even.

Since any spherical 4-system of generators of $G$ must contain an element of $G \backslash A$, the condition that $\Sigma_{1} \cap \Sigma_{2}=\{1\}$ immediately implies that $m, n$ cannot both be odd.

Assume now that $m$ is even and $n$ is odd, and consider the following map

$$
G \rightarrow(\mathbb{Z} / 2 \mathbb{Z})^{2} \text {, defined by }\left(t^{\epsilon} r^{i} s^{j}\right) \mapsto(\epsilon, i(\bmod 2)) .
$$

Note that for any $j$ and any odd $i$, one has that

$$
\left(r^{i} s^{j}\right)^{n m / 2}=\left(r^{m / 2}\right)^{n i}\left(s^{n}\right)^{m j / 2}=r^{m / 2}=: u \neq 1 .
$$

If $T=\left(t^{\epsilon_{1}} r^{i_{1}} s^{j_{1}}, t^{\epsilon_{2}} r^{i_{2}} s^{j_{2}}, t^{\epsilon_{3}} r^{i_{3}} s^{j_{3}}, t^{\epsilon_{4}} r^{i_{4}} s^{j_{4}}\right)$ is a spherical 4-system of generators of $G$, then $\epsilon_{1}+\epsilon_{2}+\epsilon_{3}+\epsilon_{4} \equiv 0(\bmod 2), i_{1}+i_{2}+i_{3}+i_{4} \equiv 0$ $(\bmod 2)$, and the images of the elements in $T$ generate $(\mathbb{Z} / 2 \mathbb{Z})^{2}$. Hence, the image in $(\mathbb{Z} / 2 \mathbb{Z})^{2}$ of such a spherical 4 -system of generators can be (up to a permutation) only one of

(i) $\{(1,1),(1,0),(0,1),(0,0)\}$,

(ii) $\{(1,1),(1,1),(1,0),(1,0)\}$, 
(iii) $\{(1,1),(1,1),(0,1),(0,1)\}$,

(iv) $\{(1,0),(1,0),(0,1),(0,1)\}$.

Therefore, for any two spherical 4 -systems $T_{1}$ and $T_{2}$ one can find $x \in T_{1}$ and $y \in T_{2}$ such that either

- $x, y \in G \backslash A$ are conjugate; or

- $x, y \in A$ and $x^{m n / 2}=u=y^{m n / 2}$;

a contradiction to $\Sigma_{1} \cap \Sigma_{2}=\{1\}$.

If both $m$ and $n$ are even, write $m=2^{\mu} m^{\prime}$ and $n=2^{\nu} n^{\prime}$, where $m^{\prime}, n^{\prime}$ are odd. Without loss of generality, we may assume that $\mu \geq \nu$.

Consider the following map

$$
G \rightarrow(\mathbb{Z} / 2 \mathbb{Z})^{3} \text {, defined by }\left(t^{\epsilon} r^{i} s^{j}\right) \mapsto(\epsilon, i(\bmod 2), j(\bmod 2)) .
$$

If $\mu>\nu$ and if $i$ is odd then

$$
\left(r^{i} s^{j}\right)^{2^{\mu-1} m^{\prime} n^{\prime}}=\left(r^{2^{\mu-1} m^{\prime}}\right)^{i n^{\prime}}\left(s^{2^{\mu-1} n^{\prime}}\right)^{j m^{\prime}}=r^{m / 2}:=u \neq 1,
$$

and if $\mu=\nu$ then

$$
\left(r^{i} s^{j}\right)^{2^{\mu-1} m^{\prime} n^{\prime}}= \begin{cases}r^{m / 2}:=u \neq 1, & \text { if } i \text { is odd and } j \text { is even; } \\ s^{n / 2}:=v \neq 1, & \text { if } i \text { is even and } j \text { is odd; } \\ r^{m / 2} s^{n / 2}:=w \neq 1, & \text { if } i, j \text { are odd. }\end{cases}
$$

If $T=\left(t^{\epsilon_{1}} r^{i_{1}} s^{j_{1}}, t^{\epsilon_{2}} r^{i_{2}} s^{j_{2}}, t^{\epsilon_{3}} r^{i_{3}} s^{j_{3}}, t^{\epsilon_{4}} r^{i_{4}} s^{j_{4}}\right)$ is a spherical 4-system of generators of $G$, then $\epsilon_{1}+\epsilon_{2}+\epsilon_{3}+\epsilon_{4} \equiv 0(\bmod 2), i_{1}+i_{2}+i_{3}+i_{4} \equiv 0$ $(\bmod 2), j_{1}+j_{2}+j_{3}+j_{4} \equiv 0(\bmod 2)$ and the images of the elements in $T$ generate $(\mathbb{Z} / 2 \mathbb{Z})^{3}$. Hence, the image in $(\mathbb{Z} / 2 \mathbb{Z})^{3}$ of such a spherical 4 -system of generators can be (up to a permutation) only one of

(i) $\{(1,1,1),(1,0,0),(0,1,0),(0,0,1)\}$,

(ii) $\{(1,1,0),(1,0,1),(0,1,0),(0,0,1)\}$,

(iii) $\{(1,1,0),(1,0,0),(0,1,1),(0,0,1)\}$,

(iv) $\{(1,1,1),(1,0,1),(0,1,1),(0,0,1)\}$,

(v) $\{(1,0,1),(1,0,0),(0,1,1),(0,1,0)\}$,

(vi) $\{(1,1,1),(1,1,0),(0,1,1),(0,1,0)\}$,

(vii) $\{(1,1,1),(1,1,0),(1,0,1),(1,0,0)\}$.

Therefore, for any two spherical 4 -systems $T_{1}$ and $T_{2}$ one can find $x \in T_{1}$ and $y \in T_{2}$ such that either

- $x, y \in G \backslash A$ are conjugate; or

- $x, y \in A$ and $x^{2^{\mu-1} m^{\prime} n^{\prime}}=u=y^{2^{\mu-1} m^{\prime} n^{\prime}}$; or

- $x, y \in A$ and $x^{2^{\mu-1} m^{\prime} n^{\prime}}=v=y^{2^{\mu-1} m^{\prime} n^{\prime}}$;

a contradiction to $\Sigma_{1} \cap \Sigma_{2}=\{1\}$.

Remark 3.28. In fact, the same argument also shows that the finite group $G=\mathbb{Z} / 2 \mathbb{Z} \ltimes(\mathbb{Z} / m \mathbb{Z} \times \mathbb{Z} / n \mathbb{Z})(m, n \in \mathbb{Z})$ cannot admit an unmixed ramification structure $\left(T_{1}, T_{2}\right)$, where $T_{1}$ has type $(2,2,2,2)$.

Recall that $G \backslash A$ contains at most four conjugacy classes, more precisely, it contains one conjugacy class if both $m, n$ are odd, two conjugacy classes if one of $m, n$ is odd and the other is even, and four if both $m, n$ are even.

Since any spherical system of generators of $G$ must contain an element of $G \backslash A$, the condition that $\Sigma_{1} \cap \Sigma_{2}=\{1\}$ immediately implies that $m, n$ cannot both be odd. 
If $m$ is even and $n$ is odd, then the above argument shows that the image in $(\mathbb{Z} / 2 \mathbb{Z})^{2}$ of any spherical system of generators contains at least two of $(1,1),(1,0),(0,1)$, and hence one can find $x \in T_{1}$ and $y \in T_{2}$ such that either $x, y \in G \backslash A$ are conjugate, or $x, y \in A$ and $x^{m n / 2}=y^{m n / 2}$, a contradiction to $\Sigma_{1} \cap \Sigma_{2}=\{1\}$.

If $m$ is even and $n$ is even, then the elements of order two in $G$ are exactly $t r^{i} s^{j}(1 \leq i \leq m, 1 \leq j \leq n), u=r^{m / 2}, v=s^{n / 2}$ and $w=r^{m / 2} s^{n / 2}$. The above argument shows that $T_{1}$ either contains four elements from four different conjugacy classes of $G \backslash A$, or two elements from two different conjugacy classes of $G \backslash A$ and two different elements of $\{u, v, w\}$. Since $T_{2}$ is also a spherical system of generators, then one can find $x \in T_{1}$ and $y \in T_{2}$ such that either $x, y \in G \backslash A$ are conjugate, or $y^{i}=x \in\{u, v, w\}$, a contradiction to $\Sigma_{1} \cap \Sigma_{2}=\{1\}$.

\section{REFERENCES}

[BC] I. Bauer, F. Catanese, Some new surfaces with $p_{g}=q=0$. Proceeding of the Fano Conference. Torino (2002), 123-142.

[BCG05] I. Bauer, F. Catanese, F. Grunewald, Beauville surfaces without real structures. In: Geometric methods in algebra and number theory, Progr. Math., vol 235, Birkhäuser Boston, (2005), 1-42.

[BCG06] I. Bauer, F. Catanese, F. Grunewald, Chebycheff and Belyi polynomials, dessins d'enfants, Beauville surfaces and group theory. Mediterr. J. Math. 3, no.2, (2006), $121-146$.

[BCG08] I. Bauer, F. Catanese, F. Grunewald, The classification of surfaces with $p_{g}=$ $q=0$ isogenous to a product. Pure Appl. Math. Q., 4, no. 2, part1, (2008), 547-586.

[B] A. Beauville, Surfaces Algébriques Complex. Astérisque 54, Paris (1978).

[Br] T. Breuer, Characters and Automorphism Group of Compact Riemann Surfaces. London Math. Soc., Lecture Notes Series 280. Cambridge University Press, (2000).

[Cat84] F. Catanese, On the moduli spaces of surfaces of general type. J. Diff. Geo. 19, (1984), 483-515.

[Cat92] F. Catanese, Chow varieties, Hilbert schemes and moduli spaces of surfaces of general type. J. Algebraic Geom. 1 (1992), no. 4, 561-595.

[Cat00] F. Catanese, Fibred surfaces, varieties isogenous to a product and related moduli spaces. Amer. J. Math. 122, (2000), 1-44.

[FMP] B. Fairbairn, K. Magaard, C. Parker, Generation of finite simple groups with an application to groups acting on Beauville surfaces, Preprint availiable at arXiv:1010.3500.

[FG] Y. Fuertes, G. González-Diez, On Beauville structures on the groups $S_{n}$ and $A_{n}$, Math. Z. 264 (2010), no. 4, 959-968.

[FGJ] Y. Fuertes, G. González-Diez, A. Jaikin-Zapirain, On Beauville surfaces, Groups Geom. Dyn. 5 (2011), 107-119.

[FJ] Y. Fuertes, G. Jones, Beauville surfaces and finite groups, J. Algebra 340 (2011) $13-27$.

[GLL] S. Garion, M. Larsen, A. Lubotzky, Beauville surfaces and finite simple groups, to appear in J. Reine Angew. Math. Preprint availiable at arXiv:1005.2316.

[GP] S. Garion, M. Penegini, New Beauville surfaces and finite simple groups. Preprint availiable at arXiv:0910.5402 v3.

[G] D. Gieseker, Global moduli for surfaces of general type. Invent. Math 43 no. 3, (1977), 233-282.

[GJT] G. Gonzales-Diez, G. Jones, D. Torres-Teigell, Beauville surfaces with abelian Beauville group. Preprint availiable at arXiv:1102.4552

[GM] R. Guralnick, G. Malle, Simple groups admit Beauville structures, Preprint availiable at arXiv:1009.6183.

[LS] M.W. Liebeck, A. Shalev, Fuchsian groups, coverings of Riemann surfaces, subgroup growth, random quotients and random walks. J. Algebra 276 (2004) 552-601. 
[Ma] A. M. Macbeath, Generators of the linear fractional groups, Number Theory (Proc. Sympos. Pure Math., Vol. XII, Houston, Tex., 1967). Amer. Math. Soc., Providence, R.I. (1969) 14-32.

[Man] M. Manetti, Iterated double covers and connected components of moduli spaces. Topology 36, (1997), 745-764.

[Su] M. Suzuki, Group Theory I. Springer-Verlag, Berlin, 1982.

[V] H. Völklein, Groups as Galois groups - an introduction. Cambridge Studies in Advanced Mathematics, 53. Cambridge University Press, Cambridge, 1996.

Shelly Garion, MaX-Planck-Institute for Mathematics, D-53111 Bonn, GerMANY

E-mail address: shellyg@mpim-bonn.mpg.de

Matteo Penegini, Lehrstuhl Mathematik ViII, Universität Bayreuth, NWII, D-95440 Bayreuth, Germany

E-mail address: matteo.penegini@uni-bayreuth.de 